\title{
Estimation of the bending rigidity and spontaneous curvature of fluid membranes in simulations
}

\author{
Hayato Shiba and Hiroshi Noguchi* \\ Institute for Solid State Physics, University of Tokyo, Chiba 277-8581, Japan
}

(Dated: October 29, 2018)

\begin{abstract}
Several numerical methods for measuring the bending rigidity and the spontaneous curvature of fluid membranes are studied using two types of meshless membrane models. The bending rigidity is estimated from the thermal undulations of planar and tubular membranes and the axial force of tubular membranes. We found a large dependence of its estimate value from the thermal undulation analysis on the upper-cutoff frequency $q_{\text {cut }}$ of the least squares fit. The inverse power-spectrum fit with an extrapolation to $q_{\text {cut }} \rightarrow 0$ yields the smallest estimation error among the investigated methods. The spontaneous curvature is estimated from the axial force of tubular membranes and the average curvature of bent membrane strips. The results of these methods show good agreement with each other.
\end{abstract}

PACS numbers: 87.16.D-, 87.17.Aa, 82.70.Uv

\section{INTRODUCTION}

When amphiphilic molecules are dissolved into aqueous environments, these molecules self-assemble into several types of characteristic structures such as spherical or cylindrical micelles, bilayers, and inverted hexagonal structures [1 4]. Among them, bilayer membrane is the basic structure of cells and organella. In living cells, biomembranes are not only static walls that separate components but also dynamical objects playing functions such as the vesicle transport of proteins via membrane fusion and fission.

On a micrometer scale, the lipid membranes can be considered as a continuum surface, where the membrane thickness can be neglected. The curvature free energy of a curved membrane is given by [5]

$$
\mathcal{F}=\int\left[\frac{\kappa}{2}\left(C_{1}+C_{2}-C_{0}\right)^{2}+\bar{\kappa} C_{1} C_{2}\right] d A
$$

where $C_{1}$ and $C_{2}$ are the principal curvatures at each position of the membrane. The coefficients $\kappa$ and $\bar{\kappa}$ are the bending rigidity and saddle-splay modulus, respectively. The spontaneous curvature $C_{0}$ vanishes when lipids symmetrically distribute in both leaflets of the bilayer. The last term in Eq. (1) is constant for a fixed topology (Gauss-Bonnet theorem). The bending rigidity and spontaneous curvature are basic quantities to understand the membrane properties. In this paper, we study the numerical measurement methods of the bending rigidity $\kappa$ and the spontaneous curvature $C_{0}$ in simulations.

Several methods have been used to measure the bending rigidity $\kappa$ in experiments and simulations. They are classified to two groups: (i) One utilizes the thermal fluctuations of the undulation modes of the membrane surface. In experiments, the surface fluctuations are mea-

*Electronic address: noguchi@issp.u-tokyo.ac.jp sured by light microscopy with vesicles, cells, etc 610]. Theoretically, the fluctuation spectrum is derived by the perturbations from planar [1, 11, 12], spherical [13 16], and cylindrical [17 19] membranes. In simulations, the fluctuation spectrum of planar membranes is widely used to measure $\kappa[12,20-25]$. The fluctuations of tubular membranes have not been simulated as yet, whereas those of quasi-spherical vesicles are calculated in Refs. 16, 26, 27]. (ii) The other utilizes force measurements. A tubular (tether) membrane is formed from a liposome by a mechanical force (induced by optical tweezers, etc.). The bending rigidity can be measured using the force strength and the surface tension of the vesicle [28 32]. The stability and the shapes of tubular membranes have been intensively studied in theories [17 19, 33-37]. In simulations, Harmandaris et al. 38 measured $\kappa$ from the axial force and radius of tubular membranes. Recently, $\kappa$ was also measured from the surface tension of the buckled membranes in simulations 39.

In living cells, biomembranes have asymmetric lipid distribution in two leaflets [40]. Such asymmetry of the membranes yields a non-zero spontaneous curvature $C_{0}$. For a closed membrane i.e. vesicle, a low flip-flop rate between the leaflets can result in an effective spontaneous curvature [15]. The vesicle morphology is varied with $C_{0}$ 41 43. Recent experiments show that the spontaneous curvature $C_{0}$ is also induced by grafting polymers, by absorption of protein onto the membrane surface, or by other means [4 48]. Since many proteins were found to control membrane curvatures, much attention has been paid to the effects of the spontaneous curvature $C_{0}$. To simulate the effects of $C_{0}$, it is important to establish numerical methods to measure $C_{0}$. In previous studies, the spontaneous curvature is estimated from the comparison of membrane shapes with the results of the continuum theory [41, 49]. In this paper, we propose two direct methods for measuring the spontaneous curvature.

Many types of membrane models have been developed for simulations from the atomistic scale to a large micrometer scale (see review articles [50 54]). Among 
them, particle-based meshless membrane models [26, 5461] are suitable for studying the large-scale membrane dynamics including topological changes such as membrane rupture, fusion, and fission. In these meshless models, a membrane particle does not represent a lipid molecule; it represents a membrane patch consisting of many molecules. The membrane particles self-assemble to form vesicles and planar membranes owing to their attractive interactions.

We employ two types of meshless models in this paper: the mls membrane model [55, 56] and a new spin membrane model. In contrast to the mls model, the spin model allows a finite spontaneous curvature $C_{0}$ similar to Yuan's meshless model [61]. In our meshelss models, the bending rigidity $\kappa$ and the line tension $\Gamma$ of the membrane edge can be varied separately for wide ranges of the fluid phase.

In Sec. III the membrane models and the simulation methods are described. The measurements of the bending rigidity $\kappa$ from the undulation mode analysis of planar membranes is explained in Sec. III. The stretching force measurement and thermal undulations of tubular membranes are described in Sec. IV] The measurement of the spontaneous curvature $C_{0}$ from the force measurement of tubular membranes and the average curvature of bent membrane strips is explained in Sec. $\mathrm{V}$ A summary is provided in Sec. VI]

\section{SIMULATION MODEL AND METHOD}

We employ two types of meshless membrane models. They use different curvature potentials. (i) The meshless mls membrane model [55, 56]: Membrane particles possess only translational degrees of freedom, and form quasi-two-dimensional structures stabilized by a mutibody potential based on moving least-squares (mls) method [55, 56]. (ii) The meshless spin membrane model: Particles also possess orientational degrees of freedom and interact with potentials similar to those described in Ref. [25]. In both the models, the bending rigidity $\kappa$ and the line tension $\Gamma$ of the membrane edge are controlled by changing the parameters of particle interactions (see Appendix). Further, the spontaneous curvature $C_{0}$ can be varied in the spin model.

\section{A. Meshless mls membrane model}

Since the model is explained in detail in Refs. 55, 56], the model is outlined only briefly in this section. A membrane consists of $N$ particles, which possess no internal degrees of freedom. The particles interact with each other via a potential

$$
\frac{U}{k_{\mathrm{B}} T}=\varepsilon\left(U_{\mathrm{rep}}+U_{\mathrm{att}}\right)+U_{\alpha},
$$

where $k_{\mathrm{B}} T$ is the thermal energy. The potential $U$ consists of a repulsive soft-core potential $U_{\text {rep }}$, an attractive potential $U_{\text {att }}$, with a coefficient $\varepsilon$, and a curvature potential $U_{\alpha}$. In a quasi-two-dimensional membrane surface, the particles interact via the potentials $U_{\text {rep }}$ and $U_{\text {att }}$. The repulsive excluded interaction potential of a diameter $\sigma$ is given by

$$
U_{\text {rep }}=\sum_{i<j} \exp \left(-20\left(r_{i j} / \sigma-1\right)+B\right) f_{\text {cut }}\left(r_{i j} / \sigma\right),
$$

where $B=0.126$, and $r_{i j}$ is the distance between particles $i$ and $j$. The diameter $\sigma$ is employed as the length unit to display the simulation results throughout this paper. A $C^{\infty}$-cutoff function

$$
f_{\text {cut }}(s)= \begin{cases}\exp \left\{a\left(1+\frac{1}{\left(|s| / s_{\text {cut }}\right)^{n}-1}\right)\right\} & \left(s<s_{\text {cut }}\right) \\ 0 & \left(s \geq s_{\text {cut }}\right)\end{cases}
$$

is employed. For Eq. (3), the values $n=12, a=1$, and $s_{\text {cut }}=1.2$ are used.

A solvent-free membrane model requires an attractive interaction mimicking the "hydrophobic" repulsion between hydrocarbon chains of lipid or surfactant molecules and aqueous solvent. We employ a potential

$$
U_{\text {att }}=\sum_{i} 0.25 \ln \left[1+\exp \left\{-4\left(\rho_{i}-\rho^{*}\right)\right\}\right]-C,
$$

which is a function of the local density of the particles $\rho_{i}$, which are defined by

$$
\rho_{i}=\sum_{j \neq i} f_{\text {cut }}\left(r_{i j} / \sigma\right) .
$$

For this attractive interaction, the values $s_{\text {half }}=1.8$ (at which $\left.f_{\text {cut }}\left(s_{\text {half }}\right)=0.5\right), s_{\text {cut }}=s_{\text {half }}+0.3$, and $n=$ 12 are used. The factor $a$ in $f_{\text {cut }}(s)$ is given by $a=$ $\ln (2)\left\{\left(s_{\text {cut }} / s_{\text {half }}\right)^{n}-1\right\} \simeq 3.715$.

The constant $C=0.25 \ln \left\{1+\exp \left(4 \rho^{*}\right)\right\} \simeq \rho^{*}$ is set to achieve $U_{\text {att }}=0$ at $\rho_{i}=0$. For $\rho_{i}<\rho^{*}$, the potential is approximately $U_{\text {att }} \simeq-\rho_{i}$, and therefore, it acts as a pair potential with $U_{\text {att }} \simeq-\sum_{i<j} 2 f_{\text {cut }}\left(r_{i j} / \sigma\right)$. For $\rho_{i}>\rho^{*}$, this function saturates to the constant $-C$. Thus, it is a pairwise potential with a smooth cutoff at the density $\rho_{i}=\rho^{*}$. We set $\rho^{*}=6$ in this paper to simulate a fluid membrane.

In addition to the potentials $U_{\text {rep }}$ and $U_{\text {att }}$, we add a curvature potential

$$
U_{\alpha}=k_{\alpha} \sum_{i} \alpha_{\mathrm{pl}}\left(\boldsymbol{r}_{i}\right)
$$

where the shape parameter "aplanarity" is defined by

$$
\alpha_{\mathrm{pl}}=\frac{9 D_{\mathrm{w}}}{T_{\mathrm{w}} M_{\mathrm{w}}}=\frac{9 \lambda_{1} \lambda_{2} \lambda_{3}}{\left(\lambda_{1}+\lambda_{2}+\lambda_{3}\right)\left(\lambda_{1} \lambda_{2}+\lambda_{2} \lambda_{3}+\lambda_{3} \lambda_{1}\right)} .
$$

The aplanarity $\alpha_{\mathrm{pl}}$ represents the degree of deviation from the planar shape, and $\lambda_{1} \leq \lambda_{2} \leq \lambda_{3}$ are the three 
eigenvalues of the weighted gyration tensor for the $i$ th particle given by $a_{\alpha \beta}=\sum_{j}\left(\alpha_{j}-\alpha_{\mathrm{G}}\right)\left(\beta_{j}-\beta_{\mathrm{G}}\right) w_{\mathrm{cv}}\left(r_{i j}\right)$, where $\alpha, \beta \in\{x, y, z\}$ and the local center of mass $\mathbf{r}_{\mathrm{G}}=\sum_{j} \mathbf{r}_{j} w_{\mathrm{cv}}\left(r_{i, j}\right) / \sum_{j} w_{\mathrm{cv}}\left(r_{i, j}\right)$. The aplanarity is calculated from three rotational invariants of the gyration tensor: the determinant $D_{\mathrm{w}}$, trace $T_{\mathrm{w}}$, and the sum of its three minors, $M_{\mathrm{w}}=a_{x x} a_{y y}+a_{y y} a_{z z}+a_{z z} a_{x x}-a_{x y}^{2}-$ $a_{y z}^{2}-a_{z x}^{2}$. This aplanarity $\alpha_{\mathrm{pl}}$ takes a value in the interval $[0,1]$ and is proportional to $\lambda_{1}$ for $\lambda_{1} \ll \lambda_{2}, \lambda_{3}$.

A Gaussian function with a $C^{\infty}$ cutoff [55] is employed for calculation of the weight of the gyration tensor,

$$
w_{\mathrm{cv}}\left(r_{i j}\right)= \begin{cases}\exp \left(\frac{\left(r_{i j} / r_{\mathrm{ga}}\right)^{2}}{\left(r_{i j} / r_{\mathrm{cc}}\right)^{n}-1}\right) & \left(r_{i j}<r_{\mathrm{cc}}\right) \\ 0 & \left(r_{i j} \geq r_{\mathrm{cc}}\right)\end{cases}
$$

which is smoothly cut off at $r_{i j}=r_{\mathrm{cc}}$. We use the parameters $n=12, r_{\mathrm{ga}}=0.5 r_{\mathrm{cc}}$, and $r_{\mathrm{cc}}=3 \sigma$ here. When the $i$ th particle has two or less particles within the cutoff distance $r_{i j}<r_{\mathrm{cc}}$, the particles could be on a plane, thereby making $\alpha_{\mathrm{pl}}=0$.

\section{B. Meshless spin membrane model}

In the meshless spin membrane model, a tilt potential $U_{\text {tilt }}$ and a bending potential $U_{\text {bend }}$ are employed instead of the aplanarity potential $U_{\alpha}: U / k_{\mathrm{B}} T=\varepsilon\left(U_{\text {rep }}+U_{\text {att }}\right)+$ $U_{\text {tilt }}+U_{\text {bend }}$. In this model, the spontaneous curvature of the membrane can be varied. Each membrane particle has one orientation unit vector $\boldsymbol{u}_{i}\left(\left|\boldsymbol{u}_{i}\right|=1\right)$. The potentials $U_{\text {tilt }}$ and $U_{\text {bend }}$ are given by

$$
\begin{aligned}
U_{\mathrm{tilt}} & =\frac{k_{\mathrm{tilt}}}{2} \sum_{i<j}\left[\left(\boldsymbol{u}_{i} \cdot \hat{\boldsymbol{r}}_{i j}\right)^{2}+\left(\boldsymbol{u}_{j} \cdot \hat{\boldsymbol{r}}_{i j}\right)^{2}\right] w_{\mathrm{cv}}\left(r_{i j}\right), \\
U_{\mathrm{bend}} & =\frac{k_{\mathrm{bend}}}{2} \sum_{i<j}\left(\boldsymbol{u}_{i}-\boldsymbol{u}_{j}-C_{\mathrm{bd}} \hat{\boldsymbol{r}}_{i j}\right)^{2} w_{\mathrm{cv}}\left(r_{i j}\right),
\end{aligned}
$$

where $\hat{\boldsymbol{r}}_{i j}=\boldsymbol{r}_{i j} / r_{i j}$. These potentials are the discretized versions of the tilt and the bending potentials of the tilt model [62, 63], respectively. The spontaneous curvature of the membrane can be controlled by the potential parameter $C_{\mathrm{bd}}$ as discussed later in Sec. V Recently, we employed similar potentials for a molecular lipid model to form bilayer membranes [25]. In the molecular model, the positions $\mathbf{r}_{i}^{\mathrm{e}}=\mathbf{r}_{i}+\mathbf{u}_{i} \sigma$ were used for calculation of the weight $w_{\mathrm{cv}}\left(r_{i j}^{\mathrm{e}}\right)$ to stabilize the bilayer structure. In contrast, here, the distance $r_{i j}$ between the centers of mass of the particles is used to calculate $w_{\mathrm{cv}}\left(r_{i j}\right)$. We use the same parameters in the functions $U_{\text {rep }}, U_{\text {att }}$, and $w_{\mathrm{cv}}\left(r_{i j}\right)$ for both the models. Unless otherwise specified, we use $\varepsilon=4$.

\section{Simulation methods: Brownian dynamics}

We simulate the membranes in the NVT ensemble (the particle number, volume, and temperature are kept con- stant). The dynamics of the membrane is calculated using Brownian dynamics (underdamped Langevin equation). The motion of the membrane particles is given by

$$
\begin{aligned}
m \frac{d^{2} \boldsymbol{r}_{i}}{d t^{2}} & =-\zeta_{\mathrm{G}} \frac{d \boldsymbol{r}_{i}}{d t}+\boldsymbol{g}_{i}^{\mathrm{G}}(t)-\frac{\partial U}{\partial \boldsymbol{r}_{i}} \\
I \frac{d \boldsymbol{\omega}_{i}}{d t} & =-\zeta_{\mathrm{r}} \boldsymbol{\omega}_{i}+\left(\mathbf{g}_{i}^{\mathrm{r}}(t)-\frac{\partial U}{\partial \mathbf{u}_{i}}\right)^{\perp}+\lambda \mathbf{u}_{i},
\end{aligned}
$$

where $m$ and $I$ are the mass and the moment of inertia of the molecule, respectively. The angular velocity $\boldsymbol{\omega}_{i}=d \mathbf{u}_{i} / d t$ is rotated by the perpendicular force $\mathbf{f}^{\perp}=\mathbf{f}-\left(\mathbf{f} \cdot \mathbf{u}_{i}\right) \mathbf{u}_{i}$. The length $\mathbf{u}_{i}^{2}=1$ is kept constant by a Lagrange multiplier $\lambda$. The friction coefficients $\zeta_{\mathrm{G}}$ and $\zeta_{\mathrm{r}}$ and the Gaussian white noises $\mathbf{g}_{i}^{\mathrm{G}}(t)$ and $\mathbf{g}_{i}^{\mathrm{r}}(t)$ obey the fluctuation-dissipation theorem;

$$
\begin{aligned}
& \left\langle g_{i, \alpha_{1}}^{\beta_{1}}(t)\right\rangle=0, \\
& \left\langle g_{i, \alpha_{1}}^{\beta_{1}}(t) g_{j, \alpha_{2}}^{\beta_{2}}\left(t^{\prime}\right)\right\rangle=2 k_{\mathrm{B}} T \zeta_{\beta_{1}} \delta_{i j} \delta_{\alpha_{1} \alpha_{2}} \delta_{\beta_{1} \beta_{2}} \delta\left(t-t^{\prime}\right) .
\end{aligned}
$$

Here, $\alpha_{1}, \alpha_{2} \in\{x, y, z\}$ and $\beta_{1}, \beta_{2} \in\{\mathrm{G}, \mathrm{r}\}$. In the following sections, we use the time unit $\tau=\zeta_{\mathrm{G}} \sigma^{2} / k_{\mathrm{B}} T$ and the energy unit $k_{\mathrm{B}} T$. We use $m=\zeta_{\mathrm{G}} \tau$ and $I=\zeta_{\mathrm{r}} \tau$. For the mls or the spin model, Eq. (11) or Eqs. (11) and (12) are integrated by the leapfrog algorithm with a time step of $\Delta t=0.005 \tau$, respectively. The simulations are performed with periodic boundary conditions in a box of dimensions $L_{x} \times L_{y} \times L_{z}$.

\section{THERMAL UNDULATIONS OF PLANAR MEMBRANES}

The undulation spectrum analysis of a planar membrane is the most widely used method to estimate the bending rigidity $\kappa$ in simulations. In this section, we compare fitting methods and establish a large dependence of the estimate value on the cutoff frequency $q_{\text {cut }}$. The spectrum of the undulation modes $\left\langle|h(q)|^{2}\right\rangle$ of the planar membranes in a Fourier space is given by [1, 12, 20]

$$
\left\langle|h(q)|^{2}\right\rangle=\frac{k_{\mathrm{B}} T}{\gamma q^{2}+\kappa q^{4}} .
$$

We calculate $|h(q)|^{2}$ for the planar membranes with $L_{x}=L_{y}$ from the raw data (the particle position $\mathbf{r}_{i}$ ) as well as from the positions averaged on a $\sqrt{N / 2} \times \sqrt{N / 2}$ square mesh with $\left(x_{\mathrm{mh}}, y_{\mathrm{mh}}\right)=\left(d_{\mathrm{mh}} n_{x}, d_{\mathrm{mh}} n_{y}\right)$. The height $z_{\mathrm{mh}}$ of a mesh point is obtained from the weighted average of the molecular position $\mathbf{r}_{i}$ in the four neighbor cells with $z_{\mathrm{mh}}=\sum_{i} z_{i} w_{\mathrm{mh}}\left(x_{i}, y_{i}\right) /\left(\sum_{i} w_{\mathrm{mh}}\left(x_{i}, y_{i}\right)\right)$ and $w_{\mathrm{mh}}\left(x_{i}, y_{i}\right)=\left(1-\left|x_{i}-x_{\mathrm{mh}}\right| / d_{\mathrm{mh}}\right)\left(1-\left|y_{i}-y_{\mathrm{mh}}\right| / d_{\mathrm{mh}}\right)$. We refer to the former and the latter spectra as the "raw spectrum" and the "mesh spectrum" respectively. Figure 1 (a) clearly shows the $q^{-4}$ dependence of the tensionless membrane (surface tension $\gamma=0$ ). For increasing $\gamma,\left\langle|h(q)|^{2}\right\rangle$ decreases at a low frequency $q$ (compare the 

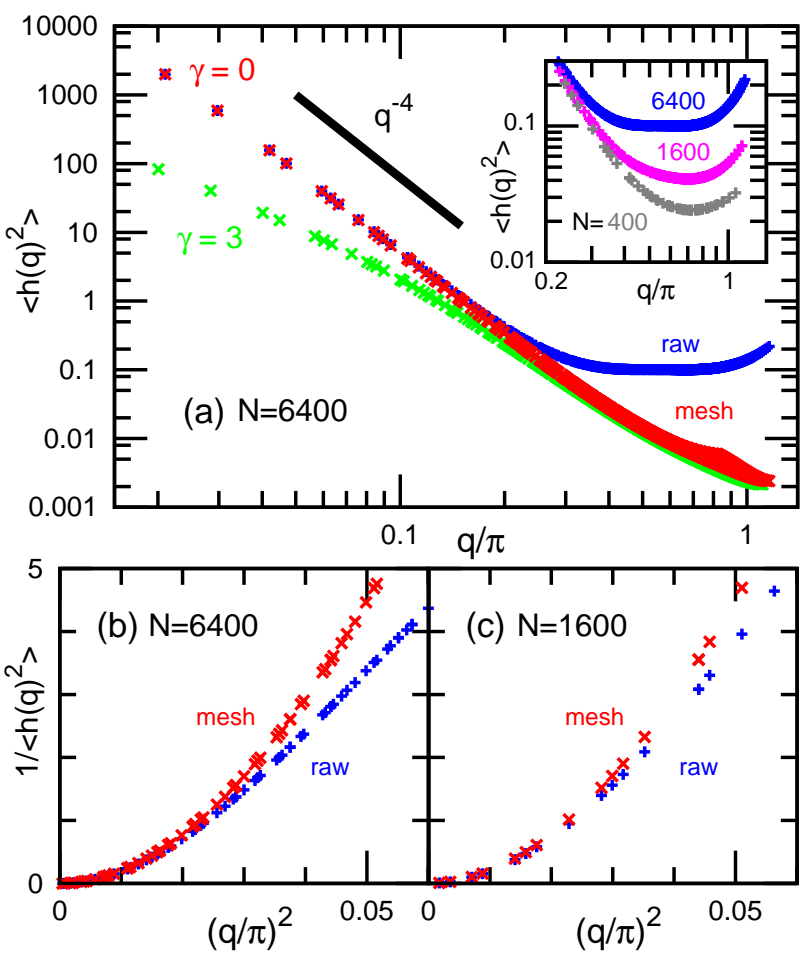

FIG. 1: (Color online) Spectra of undulation modes $\left\langle|h(q)|^{2}\right\rangle$ of a planar membranes for the mls membrane model with $k_{\alpha}=10$ and $\varepsilon=4$. Results for $\left\langle|h(q)|^{2}\right\rangle$ calculated from the particle positions (raw: + ) and from the averaged positions on a square mesh (mesh: $\times$ ) are shown. (a) Spectra for $\gamma=0$ $\left(A_{x y} / N \sigma^{2}=1.416\right)$ and $\gamma=3\left(A_{x y} / N \sigma^{2}=1.55\right)$ at the number of particles $N=6400$. The thick black line represents a slope of $q^{-4}$. The inset shows $\left\langle|h(q)|^{2}\right\rangle$ of raw data for a large $q$ at $N=400,1600$, and 6400 . The dependence of $1 /\left\langle|h(q)|^{2}\right\rangle$ on $q^{2}$ for $N=6400$ and $N=1600$ is shown in (b) and $(\mathrm{c})$, respectively.

data at $\gamma=0$ and $\gamma=3$ in Fig. 1(a)). The mesh spectrum approaches zero at a high $q$. On the contrary, the raw spectrum saturates at a finite value, which increases with increasing $N$ (see the inset of Fig. 1(a)). These high $q$ modes are caused by particle protrusion due to the short-range potential interactions between particles. Averaging over the mesh removes most of the effects of these particle protrusions at a high $q$. The effects of protrusions on bending rigidity estimation will be discussed in the last part of this section, with mls and spin models.

For the estimation of $\kappa$, two types of fits are widely used. (i) a log-log fit for the tensionless membranes,

$$
\ln \left(\left\langle|h(q)|^{2}\right\rangle\right)=-\ln \left(\frac{\kappa}{k_{\mathrm{B}} T}\right)-4 \ln (q),
$$

and (ii) an inverse power-spectrum fit,

$$
\frac{1}{\left\langle|h(q)|^{2}\right\rangle}=\frac{\gamma q^{2}+\kappa q^{4}}{k_{\mathrm{B}} T} .
$$

In the latter fit, $\gamma$ can also be estimated. We calculated both of them using the linear least squares fit for various cutoff values given by $q_{\text {cut }}$. For the fits with one fit

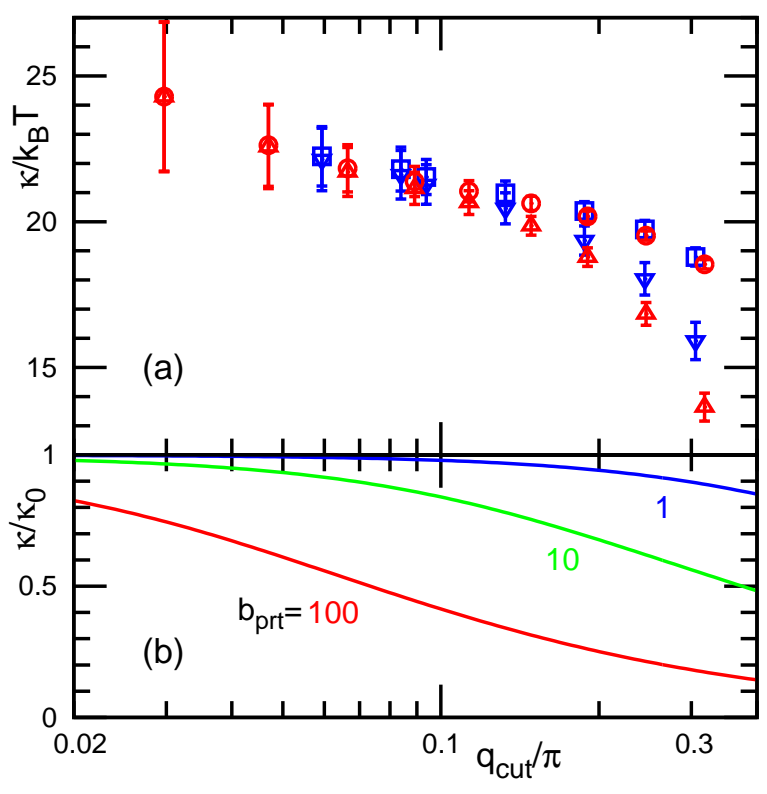

FIG. 2: (Color online) Estimation of the bending rigidity $\kappa$ of the tensionless membrane from the log-log fit for (a) the mls membrane model with $k_{\alpha}=10$ and (b) the phenomenological function Eq. (19). (a) The symbols (blue or dark gray: $\square, \nabla$ ) and (red or light gray: $\circ, \triangle$ ) represent the data for $N=$ 1600 and 6400 , respectively. The symbols at relatively lower positions $(\nabla, \triangle)$ and the symbols at relatively higher positions $(\square, \circ)$ represent the data estimated from the fits for the raw spectrum and the mesh spectrum, respectively.

parameter $(\kappa)$, the surface tension calculated from the pressure tensor is used as the value of $\gamma$. The surface tension is given by

$$
\gamma=\left\langle P_{z z}-\left(P_{x x}+P_{y y}\right) / 2\right\rangle L_{z}
$$

with the diagonal components of the pressure tensor

$$
P_{\alpha \alpha}=\left(N k_{\mathrm{B}} T-\sum_{i} \alpha_{i} \frac{\partial U}{\partial \alpha_{i}}\right) / V,
$$

where $\alpha \in\{x, y, z\}\left[64,65\right.$. In calculating $P_{\alpha \alpha}$, the periodic image $\alpha_{i}+n L_{\alpha}$ nearest to the other interacting particles is employed, when the potential interaction crosses the periodic boundary. We compare these two surface tensions, calculated from the pressure and the thermal undulations, later in this section.

Figure 2(a) shows the bending rigidity $\kappa$ estimated from the log-log fit of Eq. (15) for the data for which $q<q_{\text {cut }}$. As the cutoff frequency $q_{\text {cut }}$ increases, the estimate value of $\kappa$ gradually decreases. The fits to the mesh spectrum are less sensitive to $q_{\text {cut }}$ than those to the raw spectrum. This $q_{\text {cut }}$ dependence is caused by the neglected fluctuation modes. As shown in Fig. 11(a), $\left\langle|h(q)|^{2}\right\rangle$ of the raw spectrum deviates from $q^{-4}$ at a high $q$ because of particle protrusions. Goetz et al. reported that the protrusion modes of lipid molecules have a $q^{-2}$ dependence [12]. To clarify the influence of these protrusion modes on the spectrum, we test the least squares fit 
to a phenomenological function

$$
\left\langle|h(q)|^{2}\right\rangle=\frac{k_{\mathrm{B}} T}{\kappa_{0}}\left(\frac{1}{\left(\gamma_{0} / \kappa_{0}\right) q^{2}+q^{4}}+\frac{b_{\mathrm{prt}}}{q^{2}}\right) .
$$

The first term is the thermal-undulation mode with the bending rigidity $\kappa_{0}$ and surface tension $\gamma_{0}=0$, and the last term $\left(\propto q^{-2}\right)$ is a protrusion mode. Figure $2(b)$ shows $\kappa$ estimated by the log-log fit of Eq. (16) to the phenomenological function of Eq. (19) with $\gamma_{0}=0$ for $0.01 \pi<q<q_{\text {cut }}$. At a high $b_{\text {prt }}$ or high $q_{\text {cut }}$, the fit gives a lower $\kappa$ than the actual bending rigidity $\kappa_{0}$. It qualitatively reproduces the $q_{\text {cut }}$ dependence obtained in the simulations. A similar decrease is observed when $b_{\text {prt }} / q^{-1}$ is used instead of $b_{\text {prt }} / q^{-2}$. Thus, this dependence is not sensitive to the function shape. As a result, the fit with a lower $q_{\text {cut }}$ should yield a more accurate $\kappa$ close to $\kappa_{0}$. However, since the number of the data points for the fits is fewer for lower $q_{\text {cut }}$ and the spectrum at low $q$ has a larger statistical error, the error bar is larger in low $q_{\text {cut }}$ region. Thus, the medium cutoff $\left(q_{\text {cut }} \simeq 0.1 \pi\right.$ in Fig. 2) is a reasonable choice for the estimation of $\kappa$ from the log-log fit.

Next, we explain the inverse power-spectrum fit of Eq. (16). This fit also shows a large dependence on $q_{\text {cut }}$. Four types of fits (with one or two fit parameters for the raw and mesh spectra) for the tensionless membranes are shown in Fig. 3. The spectra are fitted with one $(\kappa)$ or two $(\kappa, \gamma)$ fit parameters. The one-parameter fit provides a lower slope of the $q_{\text {cut }}-\kappa$ curve than the twoparameter fit. The mesh spectrum gives lower slopes for $\kappa$ and $\gamma$ than the raw spectrum does (see Figs. 3(a) and (d)). Thus, substantial differences are seen between the $\kappa$ values estimated by different fits for a finite $q_{\text {cut }}$. However, all the fits converge at $q_{\text {cut }} \rightarrow 0$. A similar $q_{\text {cut }}$ dependence is observed for the fit to the phenomenological function in Eq. (19) with $\gamma_{0}=0$. At $q_{\text {cut }} \rightarrow 0, \kappa$ values converge to the correct value $\kappa_{0}$. Therefore, the bending rigidity $\kappa$ can be estimated by an extrapolation using the linear least squares fit to a straight line (see solid lines in Figs. 3(a) and (b)).

We select the extrapolated value at $q_{\text {cut }}=0$ from a one-parameter fit for the mesh spectrum as the bending rigidity $\kappa$, considering that it has the lowest slope of the $q_{\text {cut }}-\kappa$ curve. We estimate the numerical error in $\kappa$ from two contributions; i.e., for $\Delta \kappa=\Delta \kappa_{\mathrm{f} 0}+\Delta \kappa_{\mathrm{f} 1}$ : the maximum difference $\Delta \kappa_{\mathrm{fo}}$ between four extrapolated values is considered as the numerical error size from the choice of functions, and $\Delta \kappa_{\mathrm{f} 1}$ is the error of the least squares fit.

As the membrane area increases, the surface tension increases. We investigated the area dependence of $\kappa$ using the extrapolation method for the parameters $\left(k_{\alpha}=10\right.$, $\varepsilon=4$ ) investigated in our previous paper [55]. The intrinsic area $A$ of the membrane is larger than the projected area $A_{x y}$ in the $x y$ plane because of the membrane undulations. We calculate $A$ from the mesh points for the mesh spectrum. Figure 4 shows the area dependence of $\kappa$ and the difference $\Delta \gamma=\gamma_{\mathrm{fl}}-\gamma_{\mathrm{pr}}$ in the surface tension estimated from the two methods. The surface tensions $\gamma_{\mathrm{pr}}$

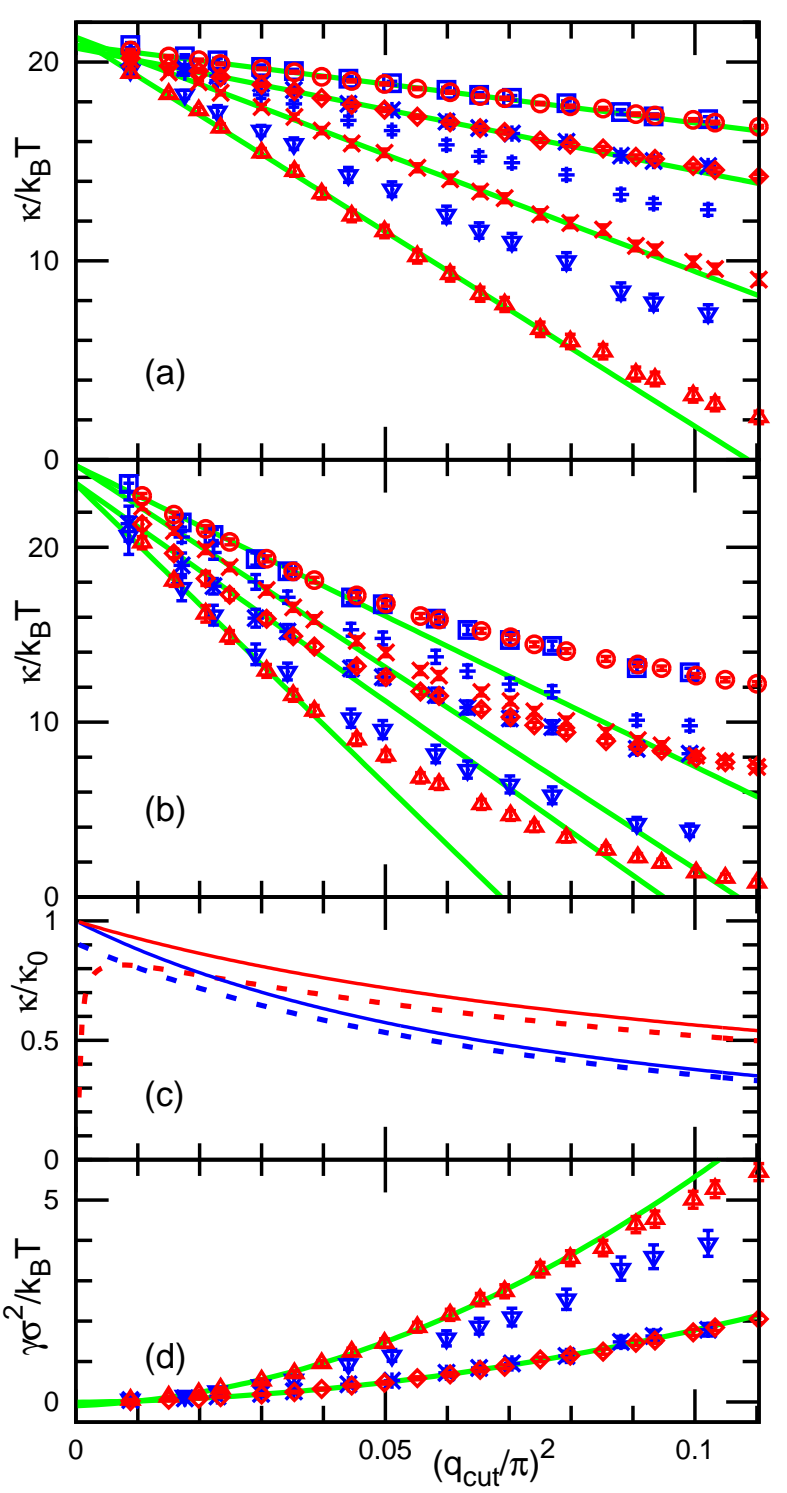

FIG. 3: (Color online) Estimation of the bending rigidity $\kappa$ from the fit to $1 /\left\langle|h(q)|^{2}\right\rangle$ with Eq. (16) for (a) the mls model with $k_{\alpha}=10$, (b) the spin model with $k_{\text {bend }}=k_{\text {tilt }}=15$ and $C_{\mathrm{bd}}=0$ at $\varepsilon=4$, and (c) the phenomenological function in Eq. (19). The symbols (blue or dark gray: $\square, *,+, \nabla$ and red or light gray: $\circ, \diamond, \times, \triangle)$ represent the data for $N=1600$ and 6400 , respectively. From the top to the bottom, the oneparameter fit for a mesh spectrum $(\square, \circ)$; two-parameter fit for a mesh spectrum $(*, \diamond)$; one-parameter fit for a raw spec$\operatorname{trum}(+, \times)$; and two-parameter fit for a raw $\operatorname{spectrum}(\nabla, \triangle)$ are shown. The solid lines in (a) and (b) are obtained by a least squares fit for the data in (a) $\left(q_{\text {cut }} / \pi\right)^{2}<0.08$ and (b) $\left(q_{\text {cut }} / \pi\right)^{2}<0.04$. (c) The solid and dashed lines represent the data for $\gamma_{0} / \kappa_{0}=0$ and 0.05 at $b_{\text {prt }}=1$, respectively. The upper or lower lines show the results obtained using one- $(\kappa)$ or two- $(\kappa, \gamma)$ parameter fits, respectively. (d) The surface tension estimated from the two-parameter fit for the mls model. The solid lines represent the curves fitted by $\gamma=\gamma_{\mathrm{fl}}+a_{\gamma} q_{\mathrm{cut}}+b_{\gamma} q_{\mathrm{cut}}^{2}$ for the data at $\left(q_{\mathrm{cut}} / \pi\right)^{2}<0.08$. 


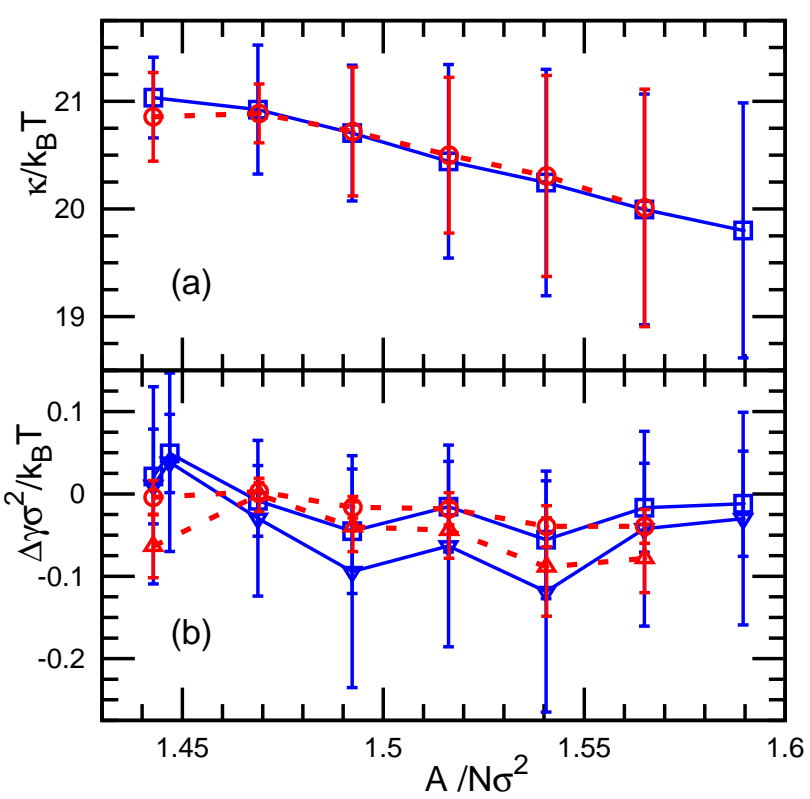

FIG. 4: (Color online) Intrinsic area $A$ dependence of (a) the bending rigidity $\kappa$ and (b) the difference $\Delta \gamma=\gamma_{\mathrm{fl}}-\gamma_{\mathrm{pr}}$ of the surface tensions estimated from the undulations (Eq. (16)) and the pressure tensor (Eq. (17)). The solid line with $(\square, \nabla)$ and dashed line with $(\circ, \triangle)$ represent the data for $N=1600$ and 6400 , respectively. (b) The symbols $(\nabla, \triangle)$ and $(\square, \circ)$ represent the data estimated from the raw spectrum and the mesh spectrum, respectively.

are estimated from the pressure tensor (Eq. (17)); $\gamma_{\mathrm{pr}}=0$ and $A / N \sigma^{2}=1.443$ at $A_{x y} / N \sigma^{2}=1.416$, whereas $\gamma_{\mathrm{pr}}=2.96$ and $A / N \sigma^{2}=1.565$ at $A_{x y} / N \sigma^{2}=1.55$. The surface tension estimated by the extrapolation with $\gamma=\gamma_{\mathrm{f}}+a_{\gamma} q_{\mathrm{cut}}+b_{\gamma} q_{\mathrm{cut}}^{2}$ has a very good agreement with $\gamma_{\mathrm{pr}}$, as shown in Fig. 4(b). Previously, Imparato reported that the surface tension estimated from the thermal undulations is smaller than that estimated from the pressure tensor in molecular simulations [66]. The reported difference may be due to the similar effects of a finite $q_{\text {cut }}$.

Although the estimated $\kappa$ decreases with an increase in $A$, it is accompanied by larger error bars. Thus, we do not find a clear dependence of $\kappa$ on $\gamma$. For a finite $\gamma_{0}$, the fit for the phenomenological function of Eq. (19) shows a deviation from the correct value $\kappa_{0}$ at $q_{\text {cut }} \rightarrow 0$ (see Fig. 3(c)). The one-parameter fit shows an abrupt decrease at $q_{\text {cut }} \simeq 0$, whereas rapid decreases are not observed in the simulations. These results suggest that systematic errors may be involved in the estimation of $\kappa$ at a finite $\gamma$. Further investigations are needed to clarify the $\kappa$ dependence on $\gamma$.

The extrapolation method works well for the spin membrane model as well as the mls membrane model. The spin model has a larger $q_{\text {cut }}$ dependence. The $q_{\text {cut }}-\kappa$ curve deviates from the straight line at $\left(q_{\text {cut }} / \pi\right)^{2} \gtrsim 0.05$ and $\left(q_{\text {cut }} / \pi\right)^{2} \gtrsim 0.1$ for the spin and mls models, respectively (compare Figs. 3(a) and (b)). This suggests

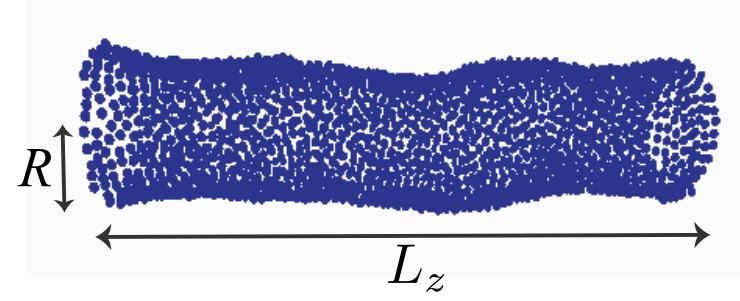

FIG. 5: (Color online) Snapshot of a tubular membrane in the simulation of the mls membrane model at $N=2400, k_{\alpha}=10$, $\varepsilon=4$, and $L_{z}=80 \sigma$.

that the spin model has greater particle protrusion than the mls model. Because the protrusion is induced by the short range interactions between particles or molecules, it is sensitive to the potentials of simulation models. To use this method, one should ensure that the spectrum at a sufficiently low $q$ is included for the linear extrapolation. The bilayer membranes of the spin molecular model 25] show a similar dependence as that of the meshless spin model (data not shown). Simulation models accompanied with larger protrusions require larger system sizes to estimate $\kappa$ from the thermal undulations.

For $\kappa$ extrapolated at $q_{\mathrm{cut}} \rightarrow 0$, no significant dependence on the system size $N$ is detected. All of the extrapolations for $N=1600$ and 6400 converge (see Figs. 3 and (4). At a finite $q$, the raw spectrum is dependent on $N$ because the protrusion amplitude increases with increasing $N$ (compare Figs. प(b) and (c)). The slope of the $q_{\text {cut }}-\kappa$ curve is higher at a larger $N$ for the raw spectrum (see Fig. 3).

We also estimated $\kappa$ using a nonlinear least squares fit for $\left\langle|h(q)|^{2}\right\rangle$ without and with the protrusion term $\sim q^{-2}$ [Eq. (14) and Eq. (19)]. Although these fits are not sensitive to $q_{\text {cut }}$, they have larger errors than the above fitting methods. Therefore, we conclude that the inverse power spectrum with an extrapolation to $q_{\text {cut }} \rightarrow 0$ is the best fitting method for the undulation of planar membranes. In the Appendix, we list the bending rigidity $\kappa$ estimated by the inverse power-spectrum fit for the mls and spin membrane models with various parameters.

\section{TUBULAR MEMBRANES WITH NO SPONTANEOUS CURVATURE}

In this section, we present the estimation of the bending rigidity $\kappa$ from tubular membranes. For a tubular membrane with a radius $R$ and a length $L_{z}$, the curvature free energy Eq. (11) is written as

$$
\mathcal{F}=2 \pi R L_{z}\left[\frac{\kappa}{2}\left(\frac{1}{R}-C_{0}\right)^{2}\right]
$$


Under the fixed area condition $A=2 \pi R L_{z}=$ const. the axial force $f_{z}=\partial F /\left.\partial L_{z}\right|_{A}$ is given by

$$
f_{z}=2 \pi \kappa\left(\frac{1}{R}-C_{0}\right) .
$$

lateral tension is anisotropic: $\gamma_{z}=f_{z} / 2 \pi R=\kappa(1 / R-$ $\left.C_{0}\right)$ in the axial direction, $\gamma_{\theta}=0$ in the azimuth direction, and $\gamma_{\mathrm{av}}=\gamma_{z} / 2$ in average. Although we assume the constant area here, the area compressibility does not change the force, Eq. (21). When the area compressibility is taken into account, the free energy Eq. (20) has an additional term $U_{\text {ar }}(A)\left(U_{\text {ar }}(A)=K_{\mathrm{A}}\left(A-A_{0}\right)^{2} / 2 A_{0}\right.$ for $A-A_{0} \ll 1$, where $A_{0}$ is the area of the tensionless membrane). Then, the same force is derived from $f_{z}=\partial F / \partial L_{z}$ and $\partial F / \partial R=0$. At $C_{0}=0$, the force is inversely proportional to $R\left(f_{z}=2 \pi \kappa / R\right)$. Using this relation, $\kappa$ was previously estimated in experiments 28 -32 and molecular simulations 38.

In this section, we investigate stretched cylindrical membranes with $C_{0}=0$ using the mls membrane model (see Fig. 5). All the tubes are connected periodically in the axial direction with the periodic length $L_{z}$. The initial conformations at each $L_{z}$ are made by slow stretching or shrinkage of the length $L_{z}$ with a speed less than $d L_{z} / d t=0.002 \sigma / \tau$. We checked that no hystereses are seen in results between stretching and shrinkage. The bending rigidity $\kappa$ is measured at fixed $L_{z}$. After discarding the data for the first calculation period $1600 \tau$, the data are averaged for a period $48000 \tau(72000 \tau)$ for $N=2400$ (1200). Eight simulations starting with independent initial conformations are performed.

Figure [6] shows the estimate values of the bending rigidity $\kappa_{\text {est }}=f_{z} R / 2 \pi$ for various $L_{z}$. The inverse radius of the tube $\sigma / R$ is employed for the horizontal axis. With increasing $\sigma / R$, the cylinder tube becomes narrower and longer. The radius $R$ of the cylinder is simply estimated by averaging the distance of each particle from the cylindrical axis: $R=\left\langle\sum_{i}\left\{\left(x_{i}-x_{\mathrm{G}}\right)^{2}+\left(y_{i}-y_{\mathrm{G}}\right)^{2}\right\}^{1 / 2} / N\right\rangle$, where $\left(x_{\mathrm{G}}, y_{\mathrm{G}}\right)$ is the center of mass of the membrane projected on the $x y$ plane. The estimate values of $\kappa_{\text {est }}$ for long tubes $(\sigma / R \gtrsim 0.06)$ have very good agreements with those obtained for planar membranes in Sec. III.

For shorter tubes $(\sigma / R \lesssim 0.06)$, large bumps (or peaks) appear at $N=1200$ (see Fig. [6(a)). Because they are suppressed at $N=2400$ (tubes that have twice the length for the same radius), these bumps are likely caused by the finite size effects in the $z$ direction. For long tubes, $\kappa_{\text {est }}$ decreases slightly with increasing $\sigma / R$. This may show the dependence of $\kappa$ on the area expansion or be on account of the higher-order terms of the bending elasticity discussed in Ref. 38. As explained later in this section, the intrinsic area $A$ is larger for longer tubes. The $\kappa_{\text {est }}$ decrease resembles that seen in the esti-

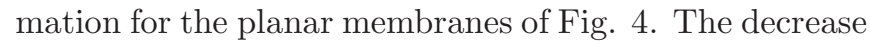
rate strongly depends on $k_{\alpha} ;-d\left(\kappa_{\text {est }} / k_{\mathrm{B}} T\right) / d(\sigma / R)=3$, 11 , and 31 for $k_{\alpha}=5,10$, and 20 , respectively. This difference can be partially explained by the effects of the thermal fluctuations. Recently, Barbetta et al. [19] de-
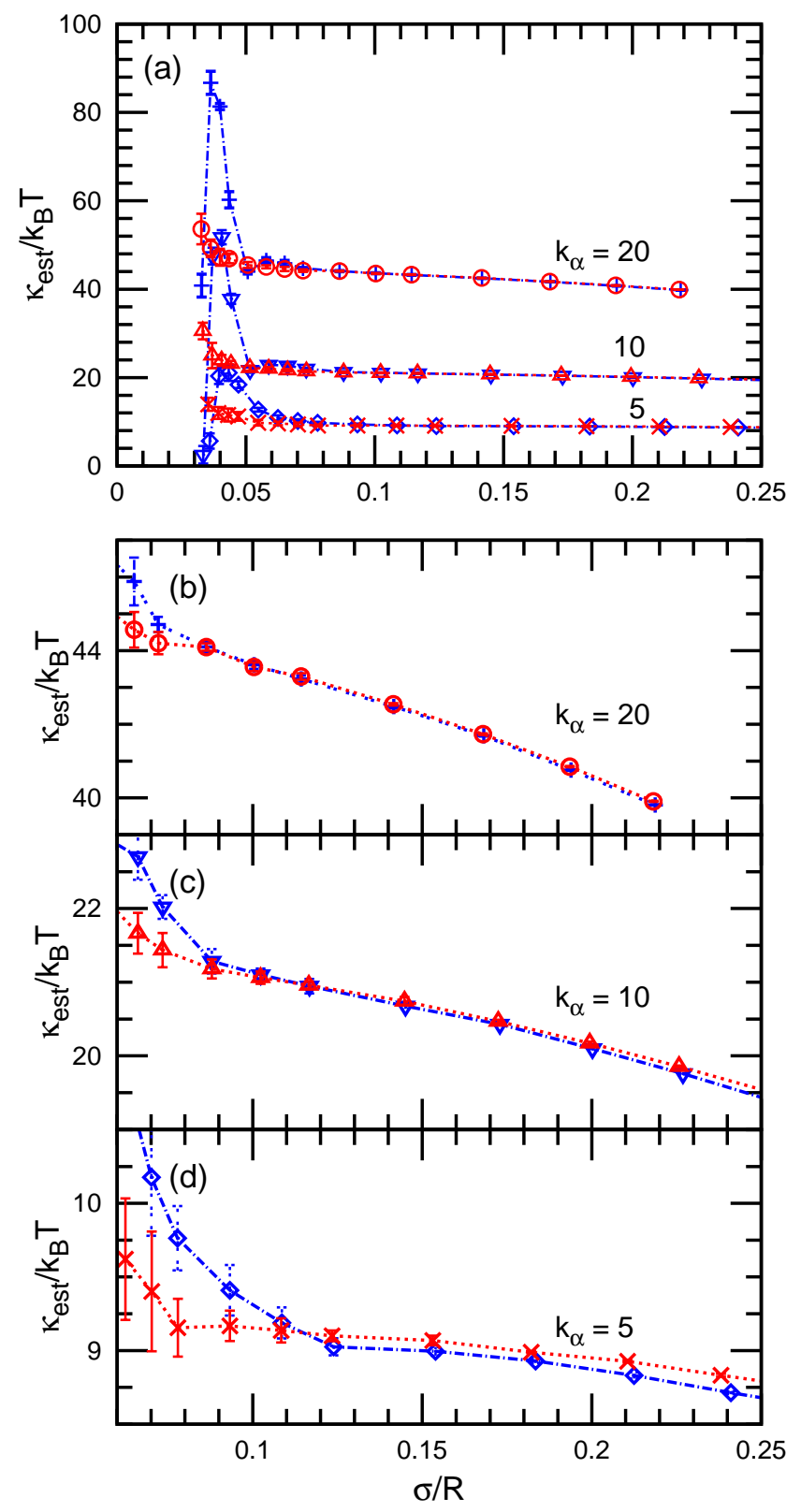

FIG. 6: (Color online) (a) Bending rigidity $\kappa_{\text {est }}=f_{z} R / 2 \pi$ estimated from the stretching force measurement using the mls membrane model. The symbols (blue or dark gray: $+, \nabla, \diamond$ and red or light gray: $\circ, \triangle, \times)$ represent the data at $N=1200$ and 2400 , respectively, for $k_{\alpha}=20(+, \circ) ; 10(\nabla, \triangle)$; and 5 $(\diamond, \times)$. (b), (c), and (d) show magnified plots of the data shown in (a) for $k_{\alpha}=20,10$, and 5 , respectively.

rived the axial force under the thermal fluctuations using perturbation theory. In their theory, the force is given by

$$
\frac{f_{z} R}{2 \pi}=\kappa-\frac{k_{\mathrm{B}} T}{2 \pi^{2}} R^{2} \Lambda^{2},
$$

where $\Lambda$ is the cutoff wave vector. For $\Lambda=1 / 2 \sigma, \kappa_{\text {est }}=$ $f_{z} R / 2 \pi$ increases by $k_{\mathrm{B}} T$ from $\sigma / R=0.1$ to 0.25 . Thus, the decrease rate for $k_{\alpha}=5$ in Fig. [6 is reduced by the thermal-fluctuation effects. 


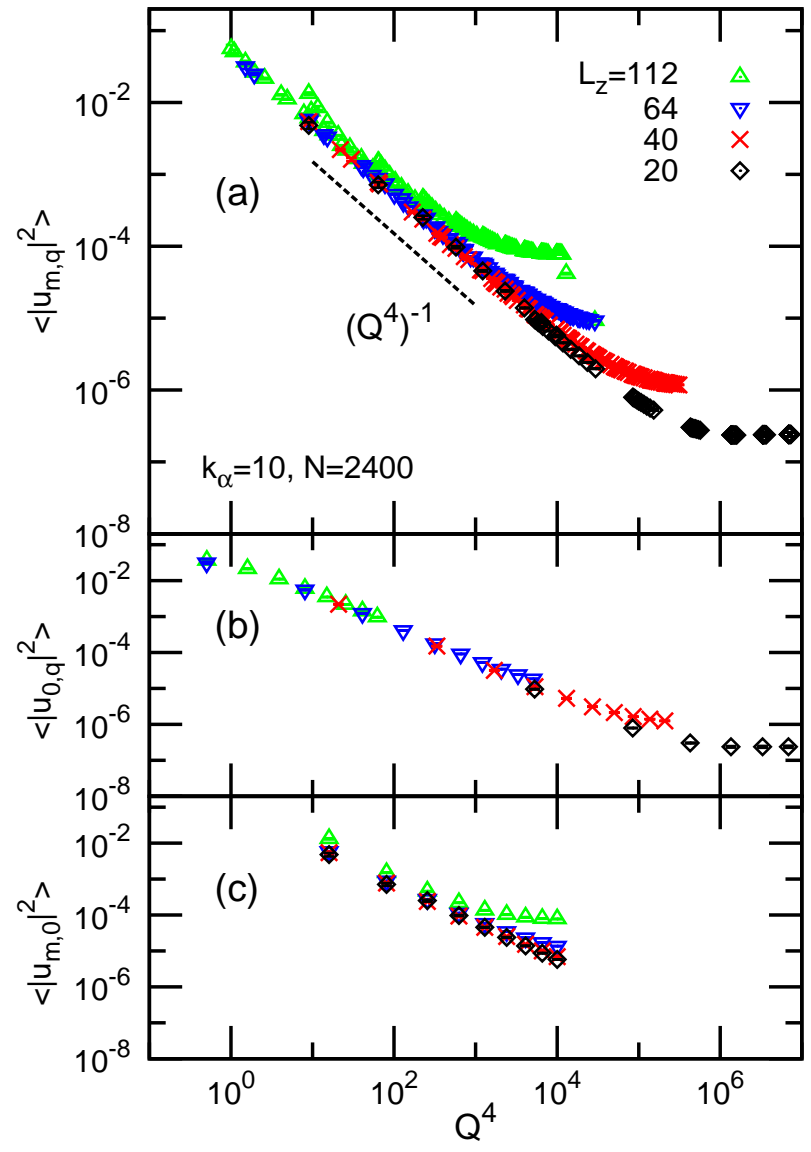

FIG. 7: (Color online) (a) Undulation spectra $\left\langle\left|u_{m, \bar{q}}\right|^{2}\right\rangle$ of the tubular membranes of the mls model at $N=2400$ and $k_{\alpha}=10$, for $L_{z} / \sigma=112(\triangle), 64(\nabla), 40(\times)$, and $20(\diamond)$. The data for $0 \leq m, n \leq 10$ are shown. The horizontal axis is the fourth power of the effective frequency $Q$ defined in Eq. (25). (b)(c) Spectra for (b) $m=0$ and $0 \leq n \leq 10$ and (c) $0 \leq m \leq 10$ and $n=0$ are extracted from the data in (a) to clearly show the $m$ and $\bar{q}$ dependence.

The bending rigidity $\kappa$ can also be estimated from the surface undulations of tubular membranes. Recently, Fournier et al. studied the thermal undulations on a cylindrical membrane theoretically [18]. They predicted the nontrivial effects of the critical Goldstone modes for narrow and long tubes. We numerically analyze the surface fluctuations and membrane area in comparison with their theoretical framework. The membrane position is expressed in the cylindrical coordinates $\boldsymbol{r}(\theta, \zeta) / R=$ $([1+u(\theta, \zeta)] \cos \theta,[1+u(\theta, \zeta)] \sin \theta, \zeta)$ for $0 \leq \theta<2 \pi$ and $0 \leq \zeta<L_{z} / R$. We calculate $u(\theta, \zeta)$ from the raw data of the particle positions $\boldsymbol{r}_{i}$. The cylindrical axis $\left(x_{\mathrm{G}}, y_{\mathrm{G}}\right)$ and the radius $R$ are estimated in the same manner as in the above force measurement. The Fourier modes of the cylindrical surface fluctuations are given by

$$
u(\theta, \zeta)=\sqrt{\frac{R}{2 \pi L_{z}}} \sum_{m, \bar{q}} u_{m, \bar{q}} e^{i(m \theta+\bar{q} \zeta)}
$$

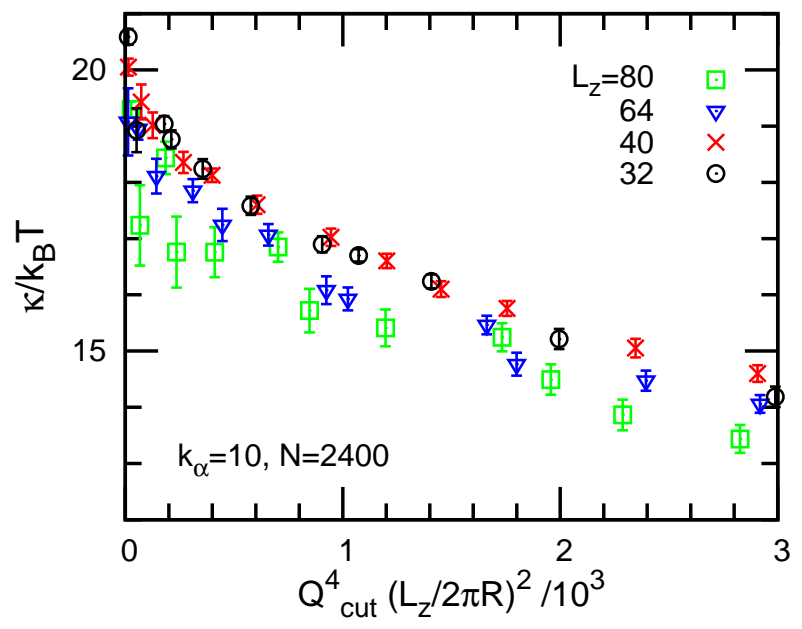

FIG. 8: (Color online) Estimation of the bending rigidity $\kappa$ for the mls membrane model from the fit to $1 /\left\langle\left|u_{m, \bar{q}}\right|^{2}\right\rangle$ with Eq. (24), for $L_{z} / \sigma=80(\square), 64(\nabla), 40(\times)$, and $32(\circ)$, for various cutoff frequencies $Q_{\text {cut }}^{4}$. The data are fitted for $0 \leq m, n \leq 10$ and $Q^{4} \leq Q_{\text {cut }}^{4}$.

where $\bar{q}=2 \pi n R / L_{z},|m| \leq 2 \pi R / l$, and $|n| \leq L_{z} / l$. The cutoff length $l$ is the mean distance between neighboring membrane particles in meshless membrane models or the membrane thickness in molecular models.

At thermal equilibrium, surface undulation of the cylindrical membrane can be estimated by the perturbation theory. The spectrum is given by [17, 18]

$$
\begin{aligned}
\left\langle\left|u_{m, \bar{q}}\right|^{2}\right\rangle & =\frac{k_{\mathrm{B}} T}{\kappa Q^{4}}, \\
Q^{4} & =\left(m^{2}-1\right)^{2}+\bar{q}^{2}\left(\bar{q}^{2}+2 m^{2}\right),
\end{aligned}
$$

where $Q$ denote the normalized amplitude of frequencies in the two-dimensional cylindrical space. Since the expression Eq. (24), for cylindrical membranes, is the counterpart of Eq. (14) for planar membranes, $\kappa$ can be estimated using a similar fitting method.

Figure 7 shows the undulation spectra for $L_{z} / \sigma=20$, 40, 64, and $112(R / \sigma=27.1,13.6,8.58$, and 5.01) at $N=1200$ and $k_{\alpha}=10$. The modes for small frequencies at $0 \leq m, n \leq 10$ are shown here $\left(\left\langle\left|u_{1,0}\right|^{2}\right\rangle\right.$ are omitted because of their divergence). While the amplitudes $\left\langle\left|u_{m, n}\right|^{2}\right\rangle$ at a low $\bar{q}$ exhibit deviations for the narrow tubes $\left(L_{z} / \sigma=112\right)$, they clearly show $Q^{-4}$ dependence at a low $Q$ when the ratio between the circumferential length and the cylinder length $2 \pi R / L_{z}$ is around unity. In these regions, $\kappa$ can be estimated by least squares fits, as explained in Sec. III

Figure 8 shows the bending rigidity $\kappa$ obtained by a linear least squares fit with various upper cutoffs $Q_{\text {cut }}^{4}$ of the inverse power spectrum $\left\langle\left|u_{m, \bar{q}}\right|^{2}\right\rangle^{-1}$ using Eq. (24), in a manner similar to that used in Sec. III. When the horizontal axis is normalized by $\left(2 \pi R / L_{z}\right)^{2}$, the data for all $L_{z}$ overlap. This dependence is very similar to that of the planar membranes shown in Fig. 3(a). $\kappa$ approaches 


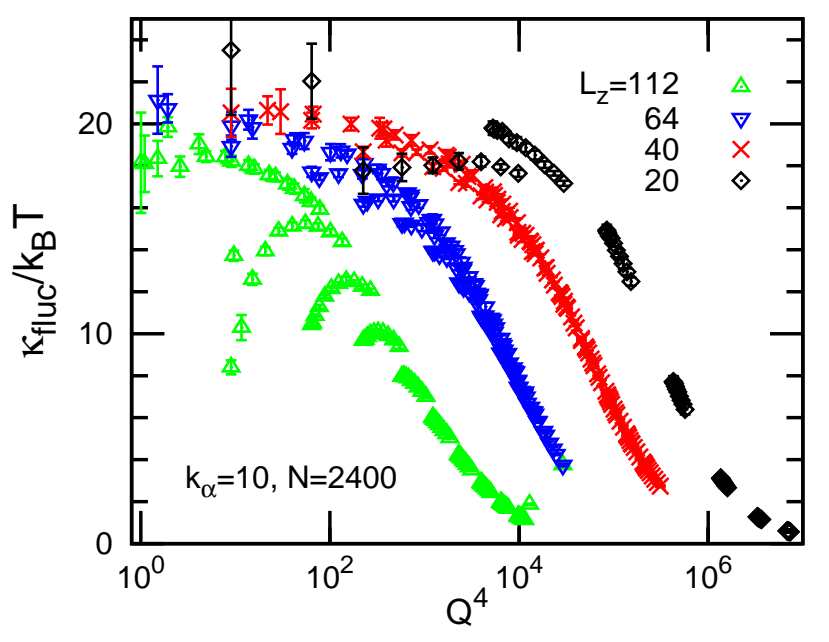

FIG. 9: (Color online) Dependence of estimate values of the bending rigidity $\kappa_{\text {fluc }}$ from the surface fluctuation spectrum on the frequency $Q^{4}$, for $L_{z} / \sigma=112(\triangle), 64(\nabla), 40(\times)$, and $20(\diamond)$, at $k_{\alpha}=10$ and $N=2400$.

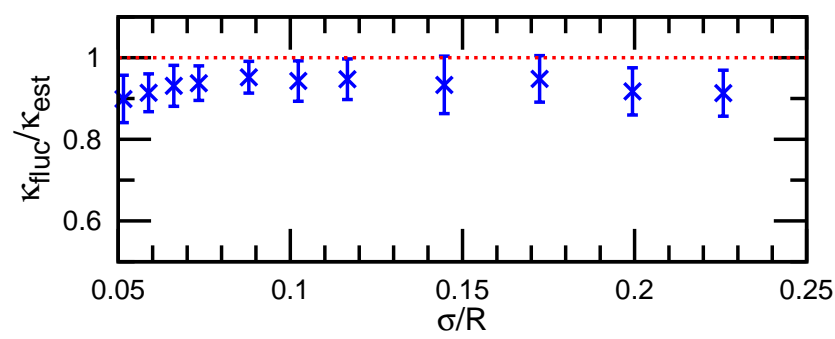

FIG. 10: (Color online) Ratio $\kappa_{\text {fluc }} / \kappa_{\text {est }}$ between the estimate values of the bending rigidity from the force measurement Eq. (21) and from the surface fluctuation spectrum shown in Fig. 9 at $k_{\alpha}=10$ and $N=2400$. The lowest ten $Q^{4}$ modes are used to estimate $\kappa_{\text {fluc }}$.

a value of around 20 as $Q_{\text {cut }} \rightarrow 0$. Thus, the spectra of $\left\langle\left|u_{m, \bar{q}}\right|^{2}\right\rangle$ well reflect the bending rigidity of the membranes.

Figure 9 shows the dependence of estimate values of the bending rigidity $\kappa_{\text {fluc }}=k_{\mathrm{B}} T /\left\langle\left|u_{m, \bar{q}}\right|^{2}\right\rangle Q^{4}$ on the frequency $\left(Q^{4}\right)$ using Eq. (24). In this figure, we plot the data for $0 \leq m, n \leq 10$. In intermediate length scales, where the radius of the tube is $0.045 \lesssim \sigma / R \lesssim 0.1$, the spectrum of $\kappa_{\text {fluc }}$ collapses into a smooth shape, whereas systematic deviations for a specific $m$ or $\bar{q}$ appear at $L_{z} / \sigma=112$ and 20 . Low $Q$ values of $\kappa_{\text {fluc }}$, which represent longer-wavelength properties, well converge to a value around $\kappa_{\text {est }}$. To compare $\kappa_{\text {fluc }}$ with $\kappa_{\text {est }}$, their ratio $\kappa_{\text {fluc }} / \kappa_{\text {est }}$ is shown in Fig. 10, In the region plotted in Fig. $10\left(15 \lesssim L_{z} / \sigma \lesssim 25\right)$, the ratio is constant $\simeq 0.9$, whereas both of them decrease with increasing $\sigma / R$. These tendencies are also obtained for other $k_{\alpha}$ or $N$. The results could be reflecting the dependence of $\kappa$ on the intrinsic area $A$ for the meshless model.

In the meshless membranes, the tubular membrane area is slightly expanded owing to the axial tension. Here,

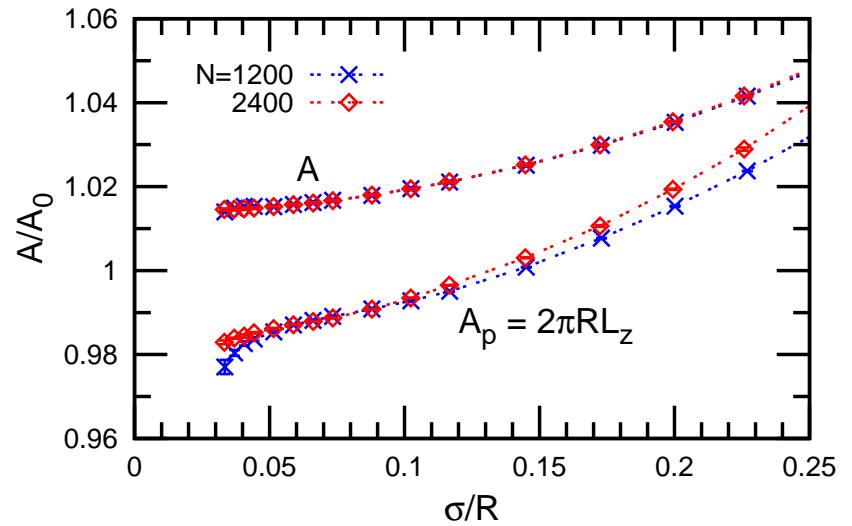

FIG. 11: (Color online) Tube radius dependence of the intrinsic membrane area $A$ and the projected area $A_{\mathrm{p}}=2 \pi R L_{z}$ at $k_{\alpha}=10$. The area is normalized by that of the tensionless planar membrane $\left(A_{0} / N \sigma^{2}=1.443\right)$. Blue (dark gray) and red (light gray) points show the results for $N=1200$ and 2400 , respectively.

we estimate the membrane area in the following manner: A Delaunay tessellation is performed for the $(\theta, \zeta)$ coordinates to construct a triangulated surface on the membrane. Then, the intrinsic membrane area $A$ is calculated as the sum over the area of the triangles for the $3 \mathrm{D}$ particle positions. Figure 11 shows the intrinsic area $A$ for $N=1200$ and 2400 in comparison with the projected area $A_{\mathrm{p}}=2 \pi R L_{z}$. As the membrane area is expanded for larger axial tension $\left(\gamma_{z}=\kappa / 2 R^{2}\right), A$ and $A_{\mathrm{p}}$ approach each other. The area expansion of $A$ is more than twice that of the planar membranes for the same average surface tension $\gamma_{\mathrm{av}}$. The anisotropy of the surface tension results in a low effective area compression modulus $K_{\mathrm{A}}$.

Fournier et al. [18] derived the dependence of the normalized excess area

$$
a_{\mathrm{ex}}=\frac{\kappa \Delta A}{k_{\mathrm{B}} T A_{\mathrm{p}}}=\frac{R}{4 \pi L_{z}} \sum_{m, \bar{q}} \frac{m^{2}+\bar{q}^{2}}{\left(m^{2}-1\right)^{2}+\bar{q}^{2}\left(\bar{q}^{2}+2 m^{2}\right)},
$$

on the axial tension $\sigma_{z}$, from the undulation spectrum Eqs. (24) and (25), where $\Delta A=A-A_{\mathrm{p}}$. They predicted that a higher axial tension generates an increase in the normalized excess area owing to the enhanced Goldstone mode fluctuations, contrary to the case for planar membranes. Figure 12 shows the $L_{z} / R$ dependence of the excess area $a_{\text {ex }}$ obtained from the perturbation theory [Eq. (26)] and the simulations. When $a_{\mathrm{ex}}$ is plotted for $L_{z} / R$, the size dependence of $a_{\mathrm{ex}}$ from Eq. (26) is seen only for the middle region of $L_{z} / R \sim 10$, and then, all the three curves converge at $L_{z} / R \rightarrow 0$ and $L_{z} / R \rightarrow \infty$. The enhanced fluctuations in the azimuth or axial direction generates a large $a_{\mathrm{ex}}$ at $L_{z} / R \rightarrow 0$ or $L_{z} / R \rightarrow \infty$, respectively. For $L_{z} / R \lesssim 10$, our simulation shows good agreement with their prediction. However, for $L_{z} / R \gtrsim 10, a_{\mathrm{ex}}$ decreases in the simulation but increases in their theory. Thus, the thermal undulations 


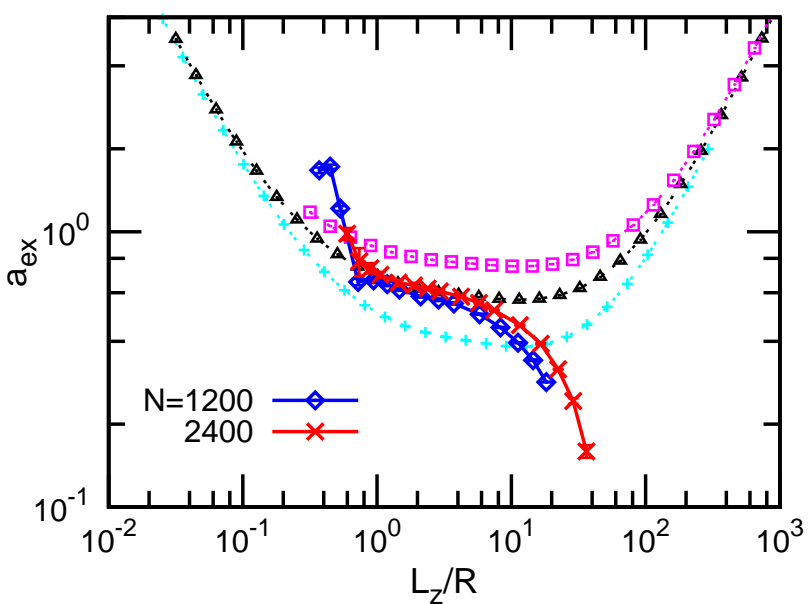

FIG. 12: (Color online) Excess area $a_{\mathrm{ex}}$ dependence on $L_{z} / R$ calculated from the simulations and the perturbation theory Eq. (26). For the simulations, $a_{\mathrm{ex}}$ is shown in the range $10 \leq L_{z} / \sigma \leq 72$ and $18 \leq L_{z} / \sigma \leq 144$ for $N=1200(\diamond)$ and $2400(\times)$ at $k_{\alpha}=10$, respectively. The analytical data from Eq. (26) are also shown for $L_{z} / l=10^{2}(+), 10^{3}(\triangle)$, and $10^{4}$ $(\square)$.

of longer tubes are suppressed in the simulations. This discrepancy may be caused by the suppression of the protrusion modes or the effects of the higher-order terms of the perturbations. Further study is necessary to clarify the origin of this difference.

When the solvent is explicitly taken into account or when bilayer membranes have a low flip-flop frequency, the bending rigidity is difficult to simulate using tubular membranes. The tubular membranes would, in such a case, exhibit very slow relaxation to the thermal equilibrium state, considering that a radius variation of the tubular membrane accompanies changes in the tube volume and in the area difference between the two leaflets. Therefore, the Laplace pressure needs to be taken into account or an additional numerical technique is required to exchange the solvent particles or lipids between the upper and the lower sides of the bilayers.

\section{MEASUREMENT OF SPONTANEOUS CURVATURE}

Next, we investigate the estimation method of the spontaneous curvature $C_{0}$ using the spin membrane model. We estimate $C_{0}$ from the axial force of tubular membranes and the shape of membrane strips. When the membranes have a nonzero spontaneous curvature, the membrane tube has the lowest free energy at $1 / R=C_{0}$, where $f_{z}$ becomes zero (see Eqs. (20) and (21)). Figure 13 shows that $1 / R-f_{z}$ lines move down for increasing $C_{\mathrm{bd}}$. The finite-size effects discussed in Sec. IV for a small $1 / R$ are also seen for a finite $C_{0}$ (See Fig. 6(a)). The spontaneous curvature $C_{0}$ and the bending rigid-

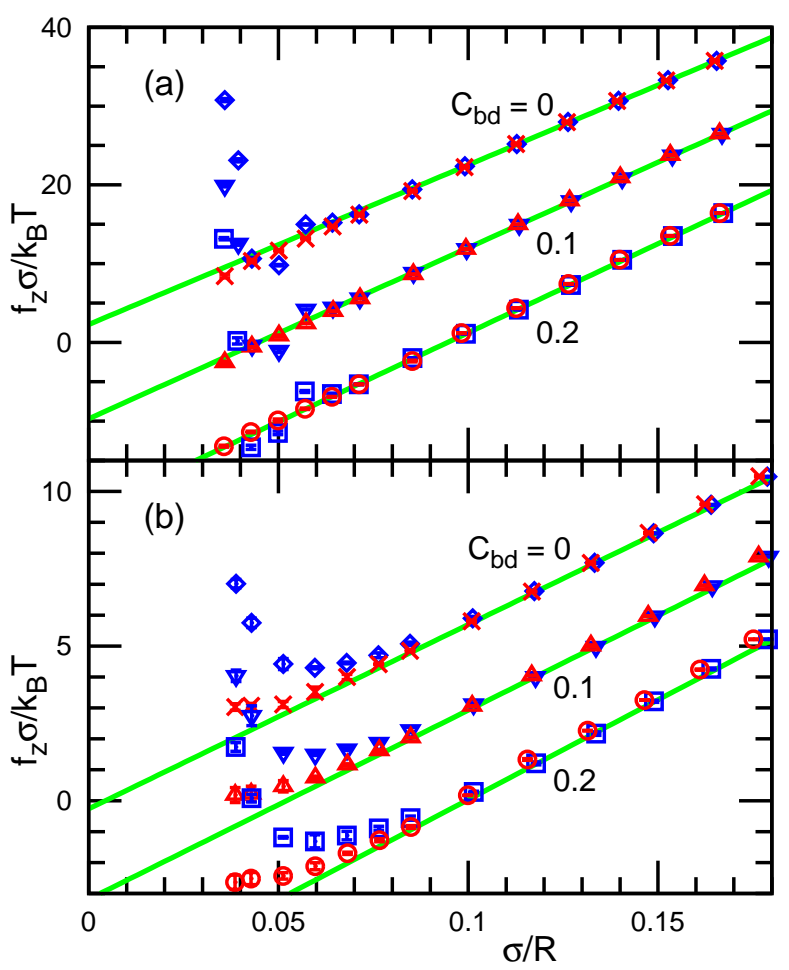

FIG. 13: (Color online) Force $f_{z}$ dependence on the radius $R$ of the membrane tube for the spin membrane model at $\varepsilon=5$ and $C_{\mathrm{bd}}=0,0.1$, and 0.2 . (a) $k_{\text {bend }}=k_{\text {tilt }}=20$. (b) $k_{\text {bend }}=k_{\text {tilt }}=5$. The symbols $(\diamond, \nabla, \square)$ and $(\times, \triangle, \circ)$ represent $N=1200$ and $N=2400$, respectively. The solid lines are obtained by linear least squares fits.

ity $\kappa$ were estimated by the linear least squares fit to Eq. (21) for $\sigma / R>0.08$ at $N=2400$. The estimated $C_{0}$ increases proportionally with $C_{\mathrm{bd}}$, as shown in Fig. 14(a): $C_{0} \sigma=0.5 C_{\mathrm{bd}}$. A small deviation $(\lesssim 0.01 / \sigma)$ from the line is almost independent of $C_{\mathrm{bd}}$ (see Fig. 14(b)); therefore, it is considered a systematic error because the symmetric membrane at $C_{\mathrm{bd}}=0$ has $C_{0}$ precisely equal to zero.

The bending rigidity $\kappa$ is independent of $C_{\mathrm{bd}}$. The results coincide with the $\kappa$ estimated from the thermal undulations of the planar membranes (see Fig. 14(c)). The two methods have a slight dependence on $C_{\mathrm{bd}}$ with opposite tendencies, but the dependences are smaller than the error bars.

Alternatively, $C_{0}$ can be estimated from the shape of a membrane strip. The membrane is connected by the periodic boundary in one $(x)$ direction, whereas it is open with edges in the other $(y)$ direction (see the snapshot in Fig. 14(a)). Because the membrane can freely bend in the $y$ direction, the mean curvature should be $C_{0}$. The flip of the orientation vector $\boldsymbol{u}_{i}$ of the membrane particles is not observed for the investigated parameters. Thus, the membranes can maintain the value of $C_{0}$ homogeneously even when the membrane has open edges. We calculated the membrane curvature using the 


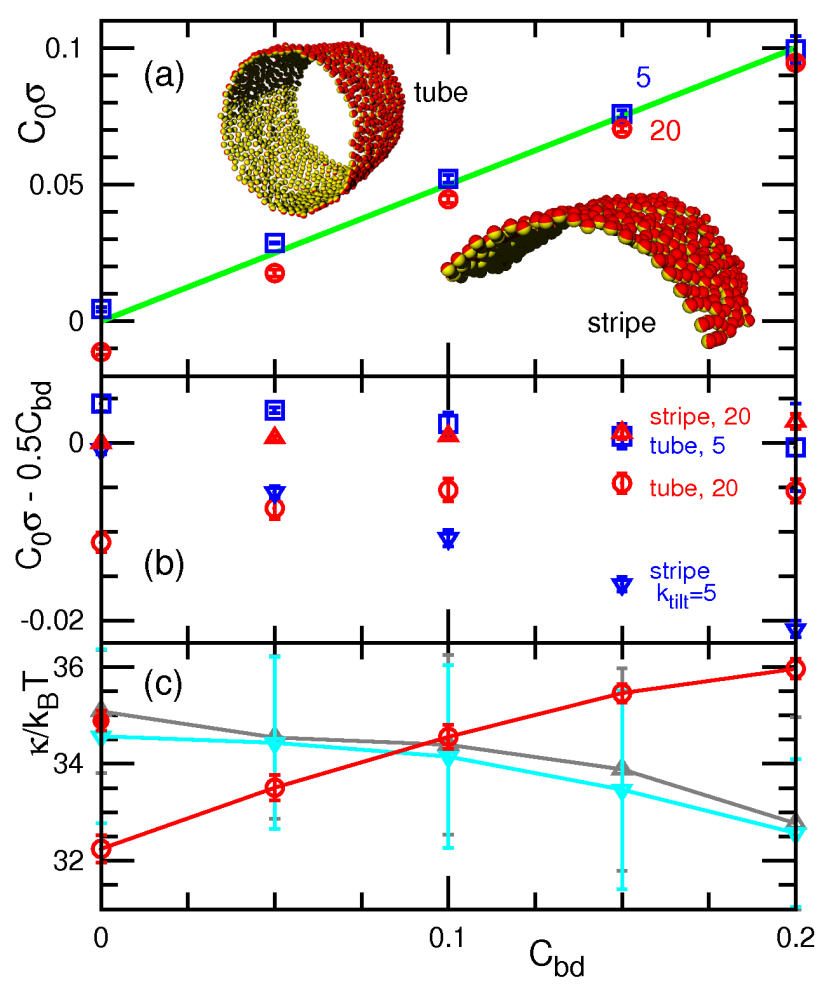

FIG. 14: (Color online) Parameter $C_{\mathrm{bd}}$ dependence of (a), (b) the spontaneous curvature $C_{0}$ and (c) the bending rigidity $\kappa$ for the spin membrane model at $\varepsilon=5$. (a) The symbols ( $\square$ ) and (o) represent $C_{0}$ obtained from the membrane tube for $k_{\text {bend }}=k_{\text {tilt }}=5$ and $k_{\text {bend }}=k_{\text {tilt }}=20$, respectively. The solid line shows the line $C_{0} \sigma=0.5 C_{\mathrm{bd}}$. The snapshots of a membrane tube and strip are shown in the inset at $N=1200$ and 400, respectively, for $L_{z}=24 \sigma, k_{\text {bend }}=k_{\text {tilt }}=20$, and $C_{\mathrm{bd}}=0.2$. (b) The symbols $(\square, \circ)$ and $(\nabla, \triangle)$ represent the relative spontaneous curvature $C_{0} \sigma-0.5 C_{\mathrm{bd}}$ obtained from the membrane tubes and membrane strips, respectively. (c) The opened or closed circles represent the values estimated by the fits with two fit parameters $\left(\kappa, C_{0}\right)$ or one parameter $(\kappa)$, respectively. The triangles $(\triangle, \nabla)$ represent $\kappa$ estimated by the undulation analysis of the planar membranes with $N=$ 1600 and 6400, respectively.

second-order moving least-squares fit [5.5] with the weight function of the potentials $w_{\mathrm{cv}}(r)$ for strips of $N=400$ with $L_{z} / \sigma=20$ to 30 . For a large bending rigidity $\kappa=34 k_{\mathrm{B}} T\left(k_{\mathrm{tilt}}=20\right)$, the resulting $C_{0}$ follows the relation $C_{0} \sigma=0.5 C_{\mathrm{bd}}$ better than that of the tube estimation. However, it seems to underestimate $C_{0}$ for a small bending rigidity $\kappa=9 k_{\mathrm{B}} T\left(k_{\text {tilt }}=5\right)$ owing to large particle protrusions. We confirmed that the resulting values were not sensitive to the shape of the weight in the mls fit. When a larger radius $\left(r_{\text {ga }}=2.5 \sigma\right.$ and $\left.r_{\mathrm{cc}}=5 \sigma\right)$ is used for the weight $w_{\mathrm{cv}}(r)$, the differences from the $C_{0}$ values calculated with the original weight are less than $5 \%$.

The bending elasticity generated by the bending and tilt potentials can be derived from the continuum theory [5] as discussed in Ref. 25]. When the orientation vec- tors $\mathbf{u}_{i}$ are equal to the normal vectors of the membrane without tilt deformation, the bending and tilt energies are given by

$$
\begin{aligned}
U_{\mathrm{cv}}=\int d A & \frac{\kappa_{\text {bend }}^{\prime}}{2}\left[\left(C_{1}-C_{0}^{\prime}\right)^{2}+\left(C_{2}-C_{0}^{\prime}\right)^{2}\right] \\
& +\frac{\kappa_{\text {tilt }}^{\prime}}{2}\left(C_{1}^{2}+C_{2}^{2}\right) \\
=\int d A & \frac{\kappa_{\text {bend }}^{\prime}+\kappa_{\text {tilt }}^{\prime}}{2}\left(C_{1}+C_{2}-C_{0}\right)^{2} \\
& -\left(\kappa_{\text {bend }}^{\prime}+\kappa_{\text {tilt }}^{\prime}\right) C_{1} C_{2}+U_{0}
\end{aligned}
$$

in the continuum limit, where $C_{1}$ and $C_{2}$ are two principal curvatures of the membrane. The first and second terms in Eq. (27) are the contributions of the bending and tilt potentials, respectively. The spontaneous curvature of the bending potential is given by $C_{0}^{\prime}=C_{\mathrm{bd}} / \bar{r}_{\mathrm{nb}}$. The nearest-neighbor distance $\bar{r}_{\mathrm{nb}} \simeq 1.15 \sigma$ is obtained from the radial distribution function. By assuming a hexagonal packing of the molecules, the bending rigidities generated by the bending and tilt potentials are estimated as $\kappa_{\text {bend }}^{\prime} / k_{\mathrm{B}} T=\sqrt{3} k_{\text {bend }} w_{\mathrm{cv}}\left(\bar{r}_{\mathrm{nb}}\right)$ and $\kappa_{\text {tilt }}^{\prime} / k_{\mathrm{B}} T=$ $\sqrt{3} k_{\mathrm{tilt}} w_{\mathrm{cv}}\left(\bar{r}_{\mathrm{nb}}\right) / 2$, respectively. The bending rigidity is given by their sum; i.e., $\kappa=\kappa_{\text {bend }}^{\prime}+\kappa_{\text {tilt }}^{\prime}$. Equation (28) gives the saddle-splay modulus $\bar{\kappa}=-\kappa$ and the spontaneous curvature $C_{0}=\left\{\kappa_{\text {bend }}^{\prime} /\left(\kappa_{\text {bend }}^{\prime}+\kappa_{\text {tilt }}^{\prime}\right)\right\} C_{\text {bd }} / \bar{r}_{\text {nb }}$ with $U_{0}=\left(\kappa_{\text {bend }}^{\prime}+\kappa_{\text {tilt }}^{\prime}\right)\left(1 / 2+\kappa_{\text {tilt }}^{\prime} / \kappa_{\text {bend }}^{\prime}\right) C_{0}^{2}$. Thus, $\kappa$ and $C_{0}$ are estimated as

$$
\begin{aligned}
\kappa & =\left(k_{\text {bend }}+0.5 k_{\text {tilt }}\right) k_{\mathrm{B}} T \\
C_{0} & =\left\{k_{\text {bend }} / 1.15 \sigma\left(k_{\text {bend }}+k_{\text {tilt }} / 2\right)\right\} C_{\text {bd }},
\end{aligned}
$$

from $w_{\mathrm{cv}}(1.15 \sigma)=0.56$. For $k_{\text {bend }}=k_{\mathrm{tilt}}, C_{0}=0.58 C_{\mathrm{bd}}$. This relation explains the simulation results very well. The $16 \%$ overestimation of the factor (0.58) may be caused by the assumption of a regular hexagonal structure for the fluid state.

Another method to estimate the spontaneous curvature $C_{0}$ in a simulation was proposed by Markvoort et al. [49]. They made a sigmoidal shape of the membranes with two domains, which have opposite spontaneous curvatures. Then, $C_{0}$ was estimated from a comparison of the membrane shape with the energy minimum curve of the continuum theory [49]. The accuracy of this method is likely to be similar to that of the curved strip since both the methods use the minimum energy shape of the membranes.

Among these three methods, the $C_{0}$ estimation for tubular membranes can be applied even for a small bending rigidity $\kappa \sim 10 k_{\mathrm{B}} T$. It would be suitable for other solvent-free models, where the membrane can freely change the tube volume or the area difference between the two leaflets in a bilayer membrane. For membranes with explicit solvents or with slow flip-flop relaxation, the other two methods, with the curved membrane strip or sigmoidal membrane, would be easier to apply. 


\section{SUMMARY}

We have investigated numerical methods for measuring the bending rigidity $\kappa$ and spontaneous curvature $C_{0}$ of fluid membranes. For planar membranes, $\kappa$ is estimated from the spectrum of the thermal undulations. It is found that estimate values show a large dependence on the upper-cutoff frequency $q_{\text {cut }}$ for the least-squares fits. Among the investigated fitting methods, the inverse power-spectrum fit with the extrapolation to $q_{\text {cut }} \rightarrow 0$ gives an accurate estimation. For tubular membranes, $\kappa$ is estimated from the stretching force and the spectrum of the thermal undulations. The estimated $\kappa$ gives a reasonable agreement with the others for all of three methods as well as for previous methods using the anisotropic surface tension of a buckled membrane [39] and the thermal undulations of quasi-spherical vesicles [55]. From a comparison of these methods, it is concluded that the inverse power-spectrum fit with the extrapolation is the best estimation method for simulations.

The excess area $a_{\text {ex }}$ of tubular membranes is also investigated. For short tubes, the calculated $a_{\mathrm{ex}}$ agrees with that obtained by Fournier et al.'s perturbation theory [18]. However, with an increasing tube length, $a_{\mathrm{ex}}$ decreases in the simulation but increases in their calculation. This difference may be caused by the finite-size effects in the simulations or the effects of the higher-order terms of the perturbation theory.

The spontaneous curvature $C_{0}$ is measured from the axial force of tubular membranes and the average curvature of bent membrane strips. Both the methods provide a reasonable estimation. The methods investigated here are also suitable for other membrane simulation models from atomistic or coarse-grained molecular models to large-scale meshless models.

\section{Acknowledgments}

The authors would like to thank W. Shinoda, T. Nakamura, T. Taniguchi, H. Wu, M. Deserno, and G. Gompper for helpful discussions and comments. The numerical calculations were partly carried out on SGI Altix ICE 8400EX at ISSP Supercomputer Center, University of Tokyo. This work is supported by KAKENHI (21740308) from the Ministry of Education, Culture, Sports, Science, and Technology of Japan.

\section{Appendix A: membrane properties}

Here, we describe the parameter dependence of the properties of the tensionless membrane for the mls and spin membrane models. Figures 15 and 16] show the dependence of five quantities on curvature parameters $\left(k_{\alpha}\right.$, $k_{\text {tilt }}$, and $\left.k_{\text {bend }}\right)$ and attraction strength $\varepsilon$, respectively. The membrane is in the fluid phase for all ranges of the parameters shown in the figures.

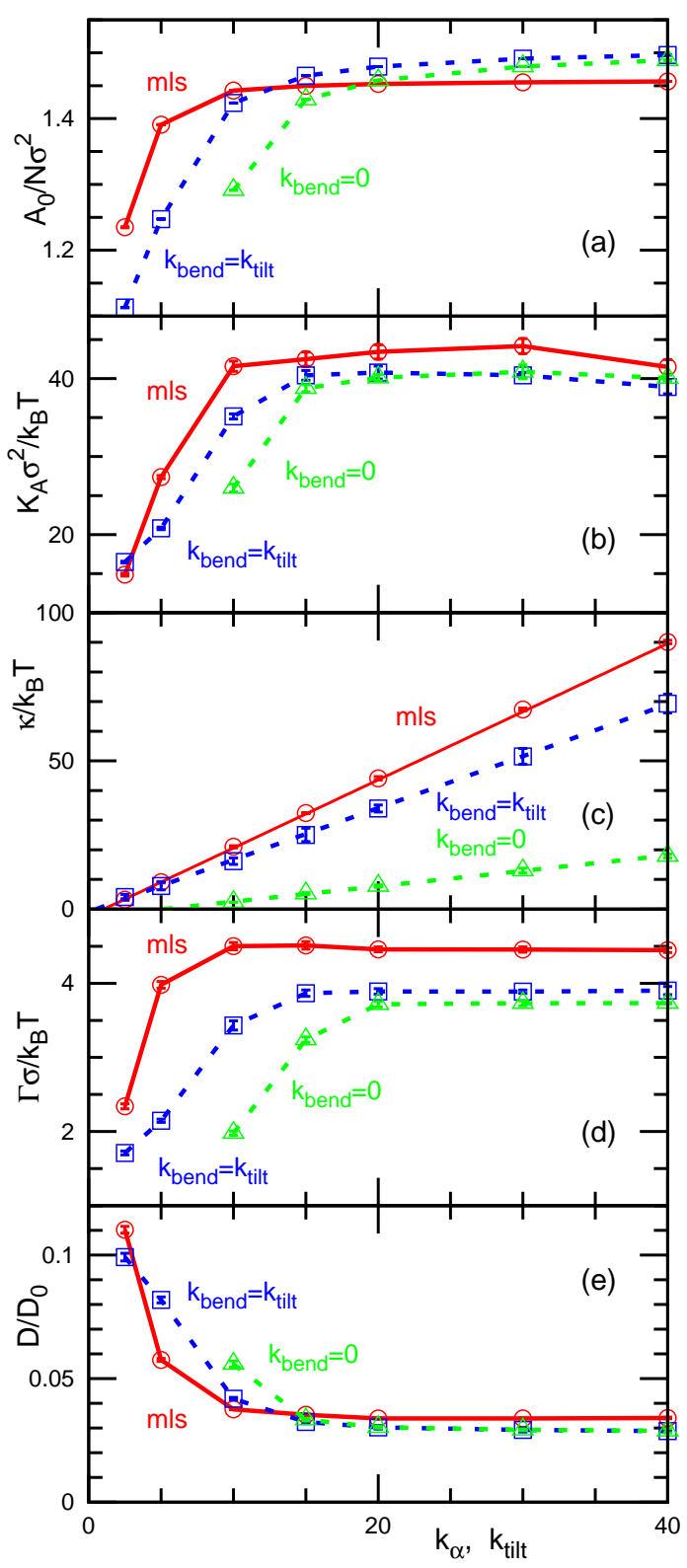

FIG. 15: (Color online) Curvature parameter dependence of (a) the intrinsic area $A_{0} / N \sigma^{2}$, (b) area compression modulus $K_{\mathrm{A}}$, (c) bending rigidity $\kappa$, (d) line tension $\Gamma$, and (e) diffusion coefficient $D$ for tensionless membranes at $\varepsilon=4$. The solid line with circles represents the data for the mls model. The dashed lines with squares and triangles represent data for the spin model at $C_{\mathrm{bd}}=0$ for $k_{\text {bend }}=k_{\text {tilt }}$ and $k_{\text {bend }}=0$, respectively. The solid and dashed lines in (c) show the linear fits for $\kappa / k_{\mathrm{B}} T=2.3 k_{\alpha}-2.4$ and $\kappa / k_{\mathrm{B}} T=1.75 k_{\mathrm{tilt}}-0.9$, as well as for $\kappa / k_{\mathrm{B}} T=0.52 k_{\mathrm{tilt}}-2.7$, respectively.

The intrinsic area $A_{0}$, the area compression modulus $K_{\mathrm{A}}$, the line tension $\Gamma$ of the membrane edge, and the particle diffusion coefficient $D$ are almost independent of the curvature parameters when they are sufficiently large $\left(k_{\alpha} \gtrsim 10\right.$ and $\left.k_{\text {tilt }} \gtrsim 15\right)$. The bending rigidity $\kappa$ is linearly dependent on the curvature param- 


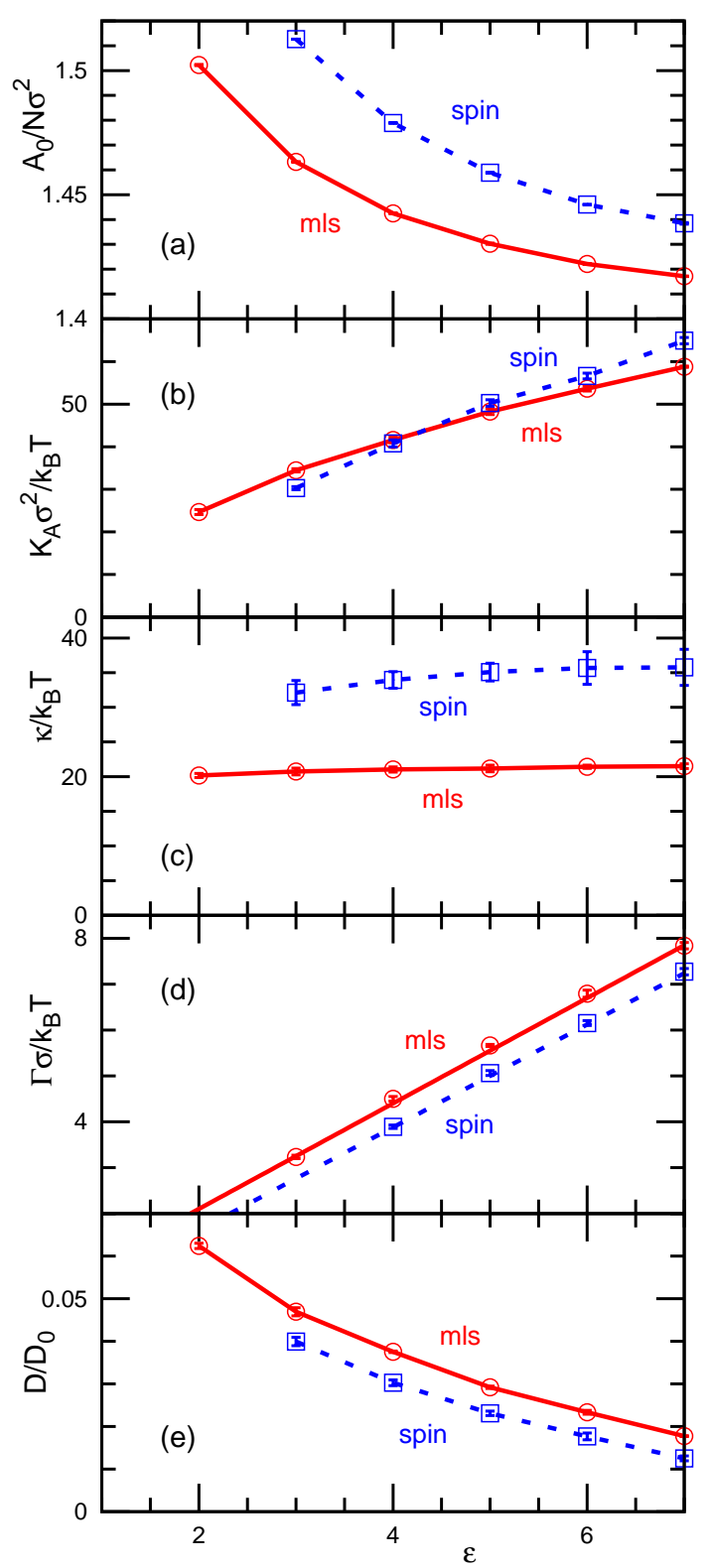

FIG. 16: (Color online) Parameter $\varepsilon$ dependence of (a) $A_{0} / N \sigma^{2}$, (b) $K_{\mathrm{A}}$, (c) $\kappa$, (d) $\Gamma$, and (e) $D$ for the tensionless membranes. The solid line with circles represents the data for the mls model at $k_{\alpha}=10$. The dashed line with squares represents the data for the spin model at $k_{\text {tilt }}=k_{\text {bend }}=20$ and $C_{\mathrm{bd}}=0$. The solid and dashed lines in (d) show the linear fits $\Gamma \sigma / k_{\mathrm{B}} T=1.15 \varepsilon-0.2$ and $\Gamma \sigma / k_{\mathrm{B}} T=1.12 \varepsilon-0.6$, respectively. eters (see Fig. 15). Thus, $\kappa$ can be varied without changing the other membrane properties. For the spin model, the dependence of $\kappa$ on curvature parameters can be quasi-quantitatively explained by Eq. (29), derived from the continuum theory. The slope is only $17 \%$ or $4 \%$ higher than the theoretical prediction for $k_{\text {bend }}=k_{\text {tilt }}$ and $k_{\text {bend }}=0$, respectively. The line tension $\Gamma$ linearly depends on $\varepsilon$, whereas $\kappa$ is almost independent of $\varepsilon$ (see Fig. 16). Thus, $\kappa$ and $\Gamma$ can be separately varied by changing the potential parameters for the spin model as well as for the mls model.

The intrinsic area $A_{0}$, the area compression modulus $K_{\mathrm{A}}=A_{0} \partial \gamma /\left.\partial A\right|_{A=A_{0}}$, and the diffusion coefficient $D$ for a tensionless membrane are calculated from planar membranes using the method explained in Ref. [55]. The unit diffusion coefficient is $D_{0}=\sigma^{2} / \tau_{0}$. The bending rigidity $\kappa$ is estimated using the extrapolation method for Eq. (16) at $N=1600$. It is $10 \%$ higher than the values estimated in our previous paper [55], where Eq. (16) is fitted with $\left(q_{\mathrm{cut}} / \pi\right)^{2}=0.05$.

The line tension $\Gamma$ of the membrane edge is calculated from the membrane strips with $N=400$, as follows: 67 69.

$$
\Gamma=\left\langle\left(P_{y y}+P_{z z}\right) / 2-P_{x x}\right\rangle L_{y} L_{z} / 2,
$$

since the length of the membrane edge is $2 L_{x}$ and $\Gamma$ is the energy per unit length of the membrane edge. The resulting $\Gamma$ values coincide with the values estimated from the membrane pore in Ref. [55].

The difference between the values is less than $0.1 k_{\mathrm{B}} T / \sigma$.
[1] S. A. Safran, Statistical Thermodynamics of Surfaces, Interfaces, and Membranes (Addison-Wesley, Reading, MA, 1994).

[2] G. Gompper and M. Schick, Self-Assembling Amphiphilic Systems, in Phase Transitions and Critical Phenomena Vol. 16 (Academic Press, London, 1994).

[3] R. Lipowsky and E. Sackmann, eds., Structure and Dy- namics of Membranes (Elsevier Science, Amsterdam, 1995).

[4] R. P. Rand, N. L. Fuller, S. M. Gruner, and V. A. Parsegian, Biochemistry 29, 76 (1990).

[5] W. Helfrich, Z. Naturforsch. C 28, 693 (1973).

[6] M. B. Schneider, J. T. Jenkins, and W. W. Webb, J. Phys. (Paris) 45, 1457 (1984). 
[7] K. Fricke, K. Wirthensohn, R. Laxhuber, and E. Sackmann, Eur. Biophys. J. 14, 67 (1986).

[8] J. F. Faucon, M. D. Mitov, P. M. Méléard, I. Bivas, and P. Bothorel, J. Phys. (Paris) 50, 2389 (1989).

[9] H. P. Duwe, J. Kaes, and E. Sackmann, J. Phys. (Paris) 51, 945 (1990).

[10] P. Méléard, C. Gerbeaud, T. Pott, L. Fernandez-Puente, I. Bivas, M. D. Mitov, J. Dufourcq, and P. Bothorel, Biophys. J. 72, 2616 (1997).

[11] E. Sackmann, FEBS Lett. 346, 3 (1994).

[12] R. Goetz, G. Gompper, and R. Lipowsky, Phys. Rev. Lett. 82, 221 (1999).

[13] W. Helfrich, J. Phys. (France) 47, 321 (1986).

[14] S. T. Milner and S. A. Safran, Phys. Rev. A 36, 4371 (1987).

[15] U. Seifert, Adv. Phys. 46, 13 (1997).

[16] G. Gompper and D. M. Kroll, in Statistical Mechanics of Membranes and Surfaces, edited by D. R. Nelson, T. Piran, and S. Weinberg (World Scientific, Singapore, 2004), 2nd ed.

[17] O.-Y. Zhong-can and W. Helfrich, Phys. Rev. A 39, 5280 (1989).

[18] J.-B. Fournier and P. Galatola, Phys. Rev. Lett. 98, 018103 (2007).

[19] C. Barbetta and J.-B. Fournier, Eur. Phys. J. E 29, 183 (2009).

[20] E. Lindahl and O. Edholm, Biophys. J. 79, 426 (2000).

[21] S. J. Marrink and A. E. Mark, J. Phys. Chem. B 105, 6122 (2001).

[22] O. Farago, J. Chem. Phys. 119, 596 (2003).

[23] J. Stecki, J. Chem. Phys. 120, 3508 (2004).

[24] E. S. Boek, J. T. Padding, W. K. den Otter, and W. J. Briels, J. Phys. Chem. B 109, 19851 (2005).

[25] H. Noguchi, J. Chem. Phys. 134, 055101 (2011).

[26] J.-M. Drouffe, A. C. Maggs, and S. Leibler, Science 254, 1353 (1991).

[27] H. Noguchi and M. Takasu, J. Chem. Phys. 115, 9547 (2001).

[28] L. Bo and R. E. Waugh, Biophys. J. 55, 509 (1989).

[29] E. Evans and W. Rawicz, Phys. Rev. Lett. 64, 2094 (1990).

[30] J. Dai and M. P. Sheetz, Biophys. J. 68, 988 (1995).

[31] E. Evans, H. Bowman, A. Leung, D. Needham, and D. Tirrell, Science 273, 933 (1996).

[32] D. Cuvelier, I. Derényi, P. Bassereau, and P. Nassoy, Biophys. J. 88, 2714 (2005).

[33] B. Božič, S. Svetina, B. Žekš, and R. E. Waugh, Biophys. J. 61, 963 (1992).

[34] T. Umeda, H. Nakajima, and H. Hotani, J. Phys. Soc. Jpn. 67, 682 (1998).

[35] D. J. Bukman, J. H. Yao, and M. Wortis, Phys. Rev. E 54, 5463 (1996).

[36] T. R. Powers, G. Huber, and R. E. Goldstein, Phys. Rev. E 65, 041901 (2002).

[37] I. Derényi, F. Jülicher, and J. Prost, Phys. Rev. Lett. 88, 238101 (2002).

[38] V. A. Harmandaris and M. Deserno, J. Chem. Phys. 125, 204905 (2006).
[39] H. Noguchi, Phys. Rev. E 83, 061919 (2011).

[40] J. A. F. O. den Kamp, Ann. Rev. Biochem. 48, 47 (1979).

[41] W. Harbich, H. J. Deuling, and W. Helfrich, J. Phys. (France) 38, 727 (1977).

[42] F. Jülicher and R. Lipowsky, Phys. Rev. E 53, 2670 (1996).

[43] S. L. Das, J. T. Jenkins, and T. Baumgart, EPL 86, 48003 (2009).

[44] T. Baumgart, B. R. Capraro, C. Zhu, and S. L. Das, Annu. Rev. Phys. Chem. 62, 483 (2011).

[45] R. Phillips, T. Ursell, P. Wiggins, and P. Sens, Nature 459, 379 (2009).

[46] J. Zimmerberg and M. M. Kozlov, Nat. Rev. Mol. Cell Biol. 7, 9 (2006).

[47] K. Akiyoshi, A. Itaya, S. M. Nomura, N. Ono, and K. Yoshikawa, FEBS Lett. 534, 33 (2003).

[48] J.-B. Fournier, N. Khalifat, N. Puff, and M. I. Angelova, Phys. Rev. Lett. 102, 018102 (2009).

[49] A. J. Markvoort, R. A. van Santen, and P. A. J. Hilbers, J. Phys. Chem. B 110, 22780 (2006).

[50] M. Müller, K. Katsov, and M. Schick, Phys. Rep. 434, 113 (2006).

[51] M. Venturoli, M. M. Sperotto, M. Kranenburg, and B. Smit, Phys. Rep. 437, 1 (2006).

[52] M. L. Klein and W. Shinoda, Science 321, 798 (2008).

[53] S. J. Marrink, A. H. de Vries, and D. P. Tieleman, Biochim. Biophys. Acta 1788, 149 (2009).

[54] H. Noguchi, J. Phys. Soc. Jpn. 78, 041007 (2009).

[55] H. Noguchi and G. Gompper, Phys. Rev. E 73, 021903 (2006).

[56] H. Noguchi and G. Gompper, J. Chem. Phys. 125, 164908 (2006).

[57] M. G. Del Pópolo and P. Ballone, J. Chem. Phys. 128, 024705 (2008).

[58] T. Kohyama, Physica A 388, 3334 (2009).

[59] P. Liu, J. Li, and Y. W. Zhang, Appl. Phys. Lett. 95, 143104 (2009).

[60] R. M. Füchslin, T. Maeke, and J. S. McCaskill, Eur. Phys. J. E 29, 431 (2009).

[61] H. Yuan, C. Huang, and S. Zhang, Soft Matter 6, 4571 (2010).

[62] M. Hamm and M. M. Kozlov, Eur. Phys. J. B 6, 519 (1998).

[63] M. Hamm and M. M. Kozlov, Eur. Phys. J. E 3, 323 (2000).

[64] J. S. Rowlinson and B. Widom, Molecular Theory of Capillarity (Clarendon, Oxford, 1982).

[65] M. P. Allen and D. J. Tildesley, Computer simulation of liquids (Oxford University Press, Oxford, 1988).

[66] A. Imparato, J. Chem. Phys. 124, 154714 (2006).

[67] T. V. Tolpekina, W. K. den Otter, and W. J. Briels, J. Chem. Phys. 121, 8014 (2004).

[68] B. J. Reynwar and M. Deserno, Biointerphases 3, FA117 (2008).

[69] A minus sign is missing on the left side of Eq. (17) in Ref. 25]. 